\title{
A First-Principles Quantum Chemical Study of Thermal Decomposition Routes for the Cationic Catalyst $\mathrm{L}_{2} \mathrm{TiMe}^{+}$
}

\author{
Tebikie Wondimagegn, Kumar Vanka, Zhitao Xu, and Tom Ziegler* \\ Department of Chemistry, University of Calgary, Calgary, Alberta, Canada T2N 1N4
}

Supporting Information Available: Optimized Cartesian coordinates $(\AA)$ for the different catalyst systems. This material is available free of charge via the Internet at http://pubs.acs.org. 
1) Methyl transfer

\section{$\left(\mathrm{CpSiMe}_{2} \mathrm{~N}^{\mathrm{t}} \mathrm{Bu}\right) \mathrm{TiMe}^{+}$}

\begin{tabular}{rrrr}
$\mathrm{Ti}$ & 0.23433 & -0.02685 & -0.19980 \\
$\mathrm{~N}$ & 2.04676 & 0.25040 & 0.05436 \\
$\mathrm{C}$ & -1.51164 & 1.54854 & -0.17707 \\
$\mathrm{C}$ & -0.28377 & 2.23070 & -0.23749 \\
$\mathrm{C}$ & 0.46455 & 1.94057 & 0.92578 \\
$\mathrm{C}$ & -0.33353 & 1.06252 & 1.70677 \\
$\mathrm{C}$ & -1.54861 & 0.83293 & 1.03290 \\
$\mathrm{H}$ & -2.29496 & 1.56233 & -0.93826 \\
$\mathrm{H}$ & 0.05761 & 2.84479 & -1.07373 \\
$\mathrm{C}$ & -0.04911 & 0.17320 & -2.23556 \\
$\mathrm{Si}$ & 2.32706 & 1.76933 & 0.89608 \\
$\mathrm{H}$ & -0.03085 & 0.60867 & 2.65618 \\
$\mathrm{H}$ & -2.37283 & 0.20176 & 1.38183 \\
$\mathrm{H}$ & -0.96522 & -0.47380 & -2.26050 \\
$\mathrm{H}$ & 0.75228 & -0.33168 & -2.80645 \\
$\mathrm{C}$ & 3.06141 & 1.54586 & 2.57494 \\
$\mathrm{C}$ & 3.13435 & 3.04284 & -0.16027 \\
$\mathrm{H}$ & 3.01810 & 2.49939 & 3.13474 \\
$\mathrm{H}$ & 2.52289 & 0.78376 & 3.16594 \\
$\mathrm{H}$ & 4.12345 & 1.24521 & 2.52439 \\
$\mathrm{H}$ & 3.04457 & 4.04168 & 0.30660 \\
$\mathrm{H}$ & 4.21155 & 2.84184 & -0.30273 \\
$\mathrm{H}$ & 2.66437 & 3.08618 & -1.15892 \\
$\mathrm{C}$ & 2.66555 & -1.01787 & -0.25452 \\
$\mathrm{C}$ & 3.44318 & -0.94157 & -1.54109 \\
$\mathrm{C}$ & 3.54644 & -1.45105 & 0.89261 \\
$\mathrm{C}$ & 1.52894 & -2.04197 & -0.38694 \\
$\mathrm{H}$ & 3.87371 & -1.92398 & -1.80381 \\
$\mathrm{H}$ & 2.80104 & -0.59988 & -2.37073 \\
$\mathrm{H}$ & 4.27240 & -0.22145 & -1.43430 \\
$\mathrm{H}$ & 3.98895 & -2.44181 & 0.68527 \\
$\mathrm{H}$ & 4.37235 & -0.73208 & 1.02749 \\
$\mathrm{H}$ & 2.97692 & -1.50841 & 1.83706 \\
$\mathrm{H}$ & 1.93080 & -3.05944 & -0.53712 \\
$\mathrm{H}$ & 0.93363 & -2.11975 & 0.56178 \\
$\mathrm{H}$ & 0.87932 & -1.88153 & -1.28257 \\
$\mathrm{H}$ & -0.30981 & 1.11488 & -2.73998 \\
& & & \\
\hline
\end{tabular}

\section{$\left(\mathrm{CpSiMe}_{2} \mathrm{~N}^{\mathrm{t}} \mathrm{Bu}\right) \mathrm{TiMe}^{+}-\mathrm{TS}$}

$\begin{array}{lrrr}\mathrm{C} & 0.24179 & -0.48645 & -0.14852 \\ \mathrm{Ti} & 2.20002 & 0.06116 & 0.04371 \\ \mathrm{~N} & 1.78724 & 1.96858 & 0.12789 \\ \mathrm{C} & 4.32269 & -0.96641 & -0.34848 \\ \mathrm{C} & 4.50361 & 0.42179 & -0.25095 \\ \mathrm{C} & 3.76329 & 1.06012 & -1.27310 \\ \mathrm{C} & 3.12817 & 0.02612 & -2.01422 \\ \mathrm{C} & 3.48272 & -1.21494 & -1.44907 \\ \mathrm{H} & 4.76287 & -1.72032 & 0.30642\end{array}$

$\begin{array}{lccc}\mathrm{H} & 5.06732 & 0.93038 & 0.53509 \\ \mathrm{C} & 2.70215 & -0.46066 & 1.98062 \\ \mathrm{i} & 2.94595 & 2.70850 & -1.01605 \\ \mathrm{H} & 2.46062 & 0.17197 & -2.86965 \\ \mathrm{H} & 3.16270 & -2.20100 & -1.80048 \\ \mathrm{H} & 2.88914 & -1.55060 & 2.05085 \\ \mathrm{H} & 1.81025 & -0.20786 & 2.58867 \\ \mathrm{C} & 2.18975 & 3.38453 & -2.55910 \\ \mathrm{C} & 3.99594 & 3.88298 & -0.07110 \\ \mathrm{H} & 2.99064 & 3.83477 & -3.17715 \\ \mathrm{H} & 1.70008 & 2.60974 & -3.17463 \\ \mathrm{H} & 1.45169 & 4.18107 & -2.35576 \\ \mathrm{H} & 4.87324 & 4.17561 & -0.67810 \\ \mathrm{H} & 3.44763 & 4.80516 & 0.19217 \\ \mathrm{H} & 4.36095 & 3.42878 & 0.86657 \\ \mathrm{C} & 0.46325 & 1.79582 & 0.20872 \\ \mathrm{C} & -0.14854 & 1.78372 & 1.55737 \\ \mathrm{C} & -0.42281 & 2.22845 & -0.90233 \\ \mathrm{H} & -1.09741 & 1.22539 & 1.58899 \\ \mathrm{H} & 0.55191 & 1.40856 & 2.31856 \\ \mathrm{H} & -0.37836 & 2.83792 & 1.81728 \\ \mathrm{H} & -1.44576 & 1.83753 & -0.78704 \\ \mathrm{H} & -0.49456 & 3.33372 & -0.86142 \\ \mathrm{H} & -0.02722 & 1.95537 & -1.89156 \\ \mathrm{H} & 0.71647 & -0.96894 & -1.05317 \\ \mathrm{H} & 0.45796 & -1.08819 & 0.77286 \\ \mathrm{H} & -0.83799 & -0.42090 & -0.32964 \\ \mathrm{H} & 3.57586 & 0.06774 & 2.39855 \\ & & & \\ & & & \end{array}$

$\left(\mathrm{CpSiMe}{ }_{2} \mathrm{~N}^{\mathrm{t}} \mathrm{Bu}\right) \mathrm{TiMe}^{+}-$Product

$\begin{array}{cccc}\mathrm{C} & 0.19598 & -0.45992 & -0.67559 \\ \mathrm{Ti} & 2.05706 & -0.08844 & 0.08431 \\ \mathrm{~N} & 2.08257 & 1.99225 & -0.06847 \\ \mathrm{C} & 3.85082 & -1.51617 & -0.37677 \\ \mathrm{C} & 4.26319 & -0.17722 & -0.47241 \\ \mathrm{C} & 3.55693 & 0.44710 & -1.53510 \\ \mathrm{C} & 2.70227 & -0.53645 & -2.08674 \\ \mathrm{C} & 2.88103 & -1.73356 & -1.37354 \\ \mathrm{H} & 4.22602 & -2.25687 & 0.33022 \\ \mathrm{H} & 4.96618 & 0.31715 & 0.20522 \\ \mathrm{C} & 2.19000 & -1.01468 & 1.89188 \\ \mathrm{Si} & 3.18948 & 2.26249 & -1.47380 \\ \mathrm{H} & 1.98900 & -0.38594 & -2.90007 \\ \mathrm{H} & 2.33834 & -2.66608 & -1.54601 \\ \mathrm{H} & 2.53879 & -2.03963 & 2.09047 \\ \mathrm{H} & 1.13984 & -0.92341 & 2.25227 \\ \mathrm{C} & 2.32983 & 2.88380 & -2.98268 \\ \mathrm{C} & 4.63752 & 3.24912 & -0.90916 \\ \mathrm{H} & 2.95884 & 2.66014 & -3.86604 \\ \mathrm{H} & 1.34967 & 2.40039 & -3.14249 \\ \mathrm{H} & 2.17764 & 3.97788 & -2.96810 \\ \mathrm{H} & 5.41130 & 3.23673 & -1.70038\end{array}$




$\begin{array}{rrrr}\mathrm{H} & 4.37968 & 4.30514 & -0.71246 \\ \mathrm{H} & 5.09011 & 2.83235 & 0.00741 \\ \mathrm{C} & 1.08612 & 2.64538 & 0.41925 \\ \mathrm{C} & 0.42205 & 2.18784 & 1.65240 \\ \mathrm{C} & 0.52000 & 3.84340 & -0.21643 \\ \mathrm{H} & -0.67288 & 2.11548 & 1.52145 \\ \mathrm{H} & 0.82415 & 1.23628 & 2.04188 \\ \mathrm{H} & 0.58704 & 2.95134 & 2.43935 \\ \mathrm{H} & -0.40695 & 3.52650 & -0.73852 \\ \mathrm{H} & 0.20275 & 4.59399 & 0.52747 \\ \mathrm{H} & 1.18549 & 4.29846 & -0.96327 \\ \mathrm{H} & -0.07918 & 0.14186 & -1.56209 \\ \mathrm{H} & -0.03329 & -1.52081 & -0.88575 \\ \mathrm{H} & -0.42339 & -0.13439 & 0.19397 \\ \mathrm{H} & 2.83517 & -0.30320 & 2.45776\end{array}$

2) $t$-butyl transfer

\section{$(\mathrm{Cp})\left(\mathrm{NC}^{t} \mathrm{Bu}_{2}\right) \mathrm{TiMe}^{+}$}

$\begin{array}{rrrr}\text { Ti } & 0.077 & 0.027 & -0.132 \\ \text { C } & 2.002 & -0.427 & 0.198 \\ \text { N } & -0.247 & 1.821 & -0.034 \\ \text { C } & -0.312 & -2.038 & -1.173 \\ \text { C } & 0.382 & -1.194 & -2.062 \\ \text { C } & -1.557 & -1.445 & -0.886 \\ \text { C } & -0.448 & -0.085 & -2.334 \\ \text { C } & -1.646 & -0.239 & -1.609 \\ \text { H } & -2.483 & 0.462 & -1.606 \\ \text { H } & 1.625 & -1.236 & 0.892 \\ \text { H } & 2.817 & -0.859 & -0.400 \\ \text { H } & 2.378 & 0.420 & 0.810 \\ \text { C } & -0.636 & 2.675 & 0.821 \\ \text { H } & -2.329 & -1.866 & -0.235 \\ \text { H } & 0.062 & -2.978 & -0.758 \\ \text { H } & 1.369 & -1.378 & -2.487 \\ \text { H } & -0.191 & 0.768 & -2.968 \\ \text { C } & -0.316 & 4.135 & 0.641 \\ \text { C } & -1.413 & 2.081 & 1.994 \\ \text { C } & -1.101 & 0.592 & 2.029 \\ \text { C } & -1.057 & 2.668 & 3.342 \\ \text { C } & -2.899 & 2.245 & 1.723 \\ \text { C } & 0.886 & 4.462 & 1.519 \\ \text { C } & 0.073 & 4.367 & -0.803 \\ \text { C } & -1.476 & 5.044 & 0.991 \\ \text { H } & -3.473 & 1.705 & 2.498 \\ \text { H } & -3.206 & 3.303 & 1.755 \\ \text { H } & -3.171 & 1.833 & 0.734 \\ \text { H } & 0.009 & 2.520 & 3.592 \\ \text { H } & -1.282 & 3.744 & 3.398 \\ \text { H } & -1.663 & 2.170 & 4.123 \\ \text { H } & -0.009 & 0.409 & 2.170 \\ \text { H } & -1.590 & 0.099 & 2.890\end{array}$

$\begin{array}{rrrr}H & -1.554 & 0.049 & 1.149 \\ H & -0.750 & 4.089 & -1.484 \\ H & 0.298 & 5.437 & -0.947 \\ H & 0.963 & 3.775 & -1.076 \\ H & -1.155 & 6.089 & 0.828 \\ H & -2.348 & 4.861 & 0.338 \\ H & -1.794 & 4.964 & 2.043 \\ H & 1.735 & 3.792 & 1.293 \\ H & 0.661 & 4.413 & 2.596 \\ H & 1.205 & 5.495 & 1.288\end{array}$

(Cp)(NC $\left.{ }^{t} \mathrm{Bu}_{2}\right) \mathrm{TiMe}^{+}-\mathrm{TS}$

$\begin{array}{lrrr}\text { Ti } & -0.000 & -0.000 & 0.000 \\ \text { C } & 2.092 & 0.000 & -0.000 \\ \text { N } & -0.343 & 1.955 & 0.002 \\ \text { C } & -0.153 & -1.771 & -1.511 \\ \text { C } & 0.604 & -0.758 & -2.127 \\ \text { C } & -1.457 & -1.271 & -1.308 \\ \text { C } & -0.225 & 0.366 & -2.287 \\ \text { C } & -1.496 & 0.052 & -1.778 \\ \text { H } & -2.348 & 0.734 & -1.726 \\ \text { H } & 2.622 & -0.916 & -0.309 \\ \text { H } & 2.391 & 0.824 & -0.678 \\ \text { H } & 2.377 & 0.281 & 1.034 \\ \text { C } & -0.192 & 0.021 & 2.292 \\ \text { C } & 0.079 & 1.992 & 1.139 \\ \text { H } & -2.298 & -1.819 & -0.874 \\ \text { H } & 0.202 & -2.772 & -1.252 \\ \text { H } & 1.644 & -0.839 & -2.444 \\ \text { H } & 0.075 & 1.338 & -2.684 \\ \text { C } & 0.630 & 3.082 & 1.998 \\ \text { C } & -0.209 & -1.348 & 1.683 \\ \text { C } & 0.845 & 0.045 & 3.366 \\ \text { C } & -1.580 & 0.410 & 2.704 \\ \text { C } & 2.116 & 2.840 & 2.192 \\ \text { C } & 0.461 & 4.340 & 1.154 \\ \text { C } & -0.090 & 3.317 & 3.308 \\ \text { H } & -1.980 & -0.321 & 3.437 \\ \text { H } & -1.621 & 1.404 & 3.176 \\ \text { H } & -2.281 & 0.425 & 1.847 \\ \text { H } & 1.836 & -0.274 & 2.996 \\ \text { H } & 0.957 & 1.021 & 3.854 \\ \text { H } & 0.549 & -0.671 & 4.161 \\ \text { H } & 0.734 & -1.614 & 1.115 \\ \text { H } & -0.284 & -2.137 & 2.454 \\ \text { H } & -1.097 & -1.540 & 1.017 \\ \text { H } & -0.603 & 4.538 & 0.944 \\ \text { H } & 0.881 & 5.194 & 1.713 \\ \text { H } & 0.993 & 4.245 & 0.193 \\ \text { H } & 0.370 & 4.203 & 3.782 \\ \text { H } & -1.156 & 3.552 & 3.139 \\ \text { H } & -0.016 & 2.489 & 4.027\end{array}$


$\begin{array}{llll}\mathrm{H} & 2.623 & 2.707 & 1.220 \\ \mathrm{H} & 2.343 & 1.974 & 2.830 \\ \mathrm{H} & 2.545 & 3.739 & 2.672\end{array}$

\section{$(\mathrm{Cp})\left(\mathrm{NC}^{\mathrm{t}} \mathrm{Bu}_{2}\right) \mathrm{TiMe}^{+}$-Product}

\begin{tabular}{|c|c|c|c|}
\hline & & & \\
\hline & & & \\
\hline & & & \\
\hline & & & \\
\hline & 0.729 & & \\
\hline & & & \\
\hline & & & \\
\hline & & & \\
\hline & & & \\
\hline & & & \\
\hline $\mathrm{H}$ & & & \\
\hline H & & & \\
\hline C & & & \\
\hline C & & & \\
\hline $\mathrm{H}$ & & $-0 . \varsigma$ & \\
\hline & & $-2 . \varepsilon$ & \\
\hline & & -1. & \\
\hline & 1. & & -2 \\
\hline & & & \\
\hline C & & -2. & \\
\hline & & $-0 .($ & \\
\hline & & & \\
\hline & 2.8 & & \\
\hline & 1.0 & & \\
\hline C & 0. & & \\
\hline H & & & \\
\hline H & & & \\
\hline $\mathrm{H}$ & -2.0 & & \\
\hline $\mathrm{H}$ & 1.4 & & \\
\hline $\mathrm{H}$ & 0.4 & & \\
\hline $\mathrm{H}$ & -0.0 & & \\
\hline $\mathrm{H}$ & -0.2 & -2 & \\
\hline H & & & \\
\hline $\mathrm{H}$ & -1.2 & -2 & \\
\hline $\mathrm{H}$ & 0.0 & & -0. \\
\hline $\mathrm{H}$ & & & \\
\hline $\mathrm{H}$ & 1.6 & & -0 . \\
\hline $\mathrm{H}$ & 1.020 & & \\
\hline $\mathrm{H}$ & -0.475 & & \\
\hline $\mathrm{H}$ & & & \\
\hline II & 3.351 & & \\
\hline II & & & \\
\hline & & & \\
\hline
\end{tabular}

\section{$(\mathrm{Cp})\left(\mathrm{NP}^{t} \mathrm{Bu}_{3}\right) \mathrm{TiMe}^{+}$}

\begin{tabular}{|c|c|c|c|}
\hline & & 00724 & \\
\hline & & & \\
\hline & & .89050 & \\
\hline & -0.69775 & .30323 & \\
\hline & & & \\
\hline & -0.71856 & & \\
\hline & -2.68240 & .38720 & \\
\hline & -2.64 & & \\
\hline & -0.38 & & \\
\hline & & & \\
\hline & -0.4 & & \\
\hline & $-0.5 \subseteq$ & & \\
\hline & & & \\
\hline & & & \\
\hline & & & \\
\hline & & & \\
\hline & & & \\
\hline & & & \\
\hline & & & \\
\hline & & & \\
\hline & & & \\
\hline & & & \\
\hline & & & \\
\hline & & & \\
\hline & & & \\
\hline & & & \\
\hline & & & \\
\hline & & & \\
\hline & -2.4 & & \\
\hline & & & \\
\hline & & & \\
\hline & & & \\
\hline & & & \\
\hline & & & \\
\hline & & & \\
\hline & & & \\
\hline & & & \\
\hline & & & \\
\hline & & & \\
\hline & & & \\
\hline & & & \\
\hline $\mathrm{H}$ & & & \\
\hline $\mathrm{H}$ & & & \\
\hline $\mathrm{H}$ & & & \\
\hline $\mathrm{H}$ & & & -3.5 \\
\hline $\mathrm{H}$ & & & \\
\hline $\mathrm{H}$ & & & \\
\hline $\mathrm{H}$ & & & $-3.494 C$ \\
\hline 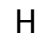 & & & \\
\hline I & & & \\
\hline & -0.65408 & 2.64205 & -4.7779 \\
\hline
\end{tabular}




$\begin{array}{rrrr}\mathrm{H} & -1.57805 & 1.14965 & -4.98770 \\ \mathrm{H} & -1.22888 & 1.76451 & -3.34490 \\ \mathrm{H} & 2.54452 & 1.44241 & -3.63183 \\ \mathrm{H} & 1.48477 & 2.79271 & -4.05648 \\ \mathrm{H} & 1.22215 & 1.90976 & -2.53474\end{array}$

\section{$(\mathrm{Cp})\left(\mathrm{NP}^{t} \mathrm{Bu}_{3}\right) \mathrm{TiMe}^{+}-\mathrm{TS}$}

$\begin{array}{lrrr}\mathrm{Ti} & 0.106198 & -0.059347 & 0.012993 \\ \mathrm{C} & 2.466850 & -0.057921 & -0.095171 \\ \mathrm{P} & 0.806118 & 2.719428 & 0.022206 \\ \mathrm{C} & -0.194285 & -0.813733 & -2.234909 \\ \mathrm{C} & 0.045381 & -1.982709 & -1.475632 \\ \mathrm{C} & -1.017386 & -2.132183 & -0.550178 \\ \mathrm{C} & -1.924582 & -1.067445 & -0.756743 \\ \mathrm{C} & -1.420681 & -0.253496 & -1.797519 \\ \mathrm{H} & 0.428459 & -0.437974 & -3.040387 \\ \mathrm{H} & 0.884276 & -2.661277 & -1.592470 \\ \mathrm{H} & -1.128876 & -2.932393 & 0.174521 \\ \mathrm{H} & -2.832700 & -0.888007 & -0.188279 \\ \mathrm{H} & -1.889480 & 0.647595 & -2.179798 \\ \mathrm{C} & -0.634405 & -0.544231 & 1.965261 \\ \mathrm{H} & -1.613107 & -0.043138 & 2.033044 \\ \mathrm{H} & -0.751787 & -1.592764 & 2.271034 \\ \mathrm{H} & 0.062708 & -0.049393 & 2.665339 \\ \mathrm{~N} & -0.430159 & 1.772004 & 0.066022 \\ \mathrm{C} & 1.294641 & 3.554767 & -1.616111 \\ \mathrm{C} & 1.061379 & 3.743365 & 1.601880 \\ \mathrm{C} & 2.813919 & 3.685244 & -1.774845 \\ \mathrm{C} & 0.712183 & 2.688911 & -2.731231 \\ \mathrm{C} & 0.642784 & 4.949133 & -1.650338 \\ \mathrm{C} & 2.388199 & 4.499907 & 1.630092 \\ \mathrm{C} & -0.132016 & 4.722266 & 1.654413 \\ \mathrm{C} & 0.941573 & 2.790789 & 2.795176 \\ \mathrm{C} & 3.060633 & -0.318737 & -1.468641 \\ \mathrm{C} & 3.397653 & 0.859293 & 0.701312 \\ \mathrm{C} & 2.186377 & -1.322107 & 0.701059 \\ \mathrm{H} & 3.006519 & 4.206197 & -2.726085 \\ \mathrm{H} & 3.278561 & 4.277688 & -0.977973 \\ \mathrm{H} & 3.309991 & 2.709251 & -1.828042 \\ \mathrm{H} & -1.092500 & 4.190845 & 1.620042 \\ \mathrm{H} & -0.107034 & 5.479017 & 0.862696 \\ \mathrm{H} & -0.069189 & 5.249523 & 2.620162 \\ \mathrm{H} & 0.954642 & 3.399503 & 3.712323 \\ \mathrm{H} & -0.001770 & 2.232904 & 2.770389 \\ \mathrm{H} & 1.778508 & 2.085118 & 2.855953 \\ \mathrm{H} & 2.446061 & 5.043097 & 2.586653 \\ \mathrm{H} & 3.255715 & 3.828940 & 1.580243 \\ \mathrm{H} & 2.463717 & 5.248373 & 0.831824 \\ \mathrm{H} & 4.097505 & -0.690040 & -1.344423 \\ \mathrm{H} & 2.515324 & -1.069328 & -2.050337 \\ \mathrm{H} & 3.130116 & 0.593869 & -2.075063 \\ \mathrm{H} & 4.362974 & 0.337893 & 0.852601 \\ & 3.626876 & 1.794759 & 0.174774\end{array}$

$\begin{array}{rrrr}\mathrm{H} & 3.010831 & 1.098227 & 1.698572 \\ \mathrm{H} & 2.709253 & -1.311223 & 1.670123 \\ \mathrm{H} & 1.120804 & -1.497611 & 1.037342 \\ \mathrm{H} & 2.450786 & -2.232955 & 0.146958 \\ \mathrm{H} & 0.972601 & 3.154796 & -3.694217 \\ \mathrm{H} & 1.132759 & 1.677185 & -2.718121 \\ \mathrm{H} & -0.380095 & 2.618702 & -2.664164 \\ \mathrm{H} & 1.090061 & 5.641404 & -0.927133 \\ \mathrm{H} & 0.825048 & 5.364004 & -2.655032 \\ \mathrm{H} & -0.444292 & 4.910464 & -1.498361\end{array}$

\section{$(\mathrm{Cp})\left(\mathrm{NP}^{t} \mathrm{Bu}_{3}\right) \mathrm{TiMe}^{+}$-Product}

$\begin{array}{lrrr}\mathrm{Ti} & -0.045347 & 0.013564 & -0.030449 \\ \mathrm{C} & 2.233116 & -0.150094 & 0.574785 \\ \mathrm{C} & 1.994599 & 1.231508 & 0.384896 \\ \mathrm{C} & 1.067201 & 1.657128 & 1.361876 \\ \mathrm{C} & 0.726391 & 0.542148 & 2.159580 \\ \mathrm{C} & 1.444930 & -0.578576 & 1.672171 \\ \mathrm{H} & 2.899267 & -0.766720 & -0.022968 \\ \mathrm{H} & 2.453300 & 1.855683 & -0.374903 \\ \mathrm{H} & 0.668008 & 2.662150 & 1.467012 \\ \mathrm{H} & 0.053814 & 0.547458 & 3.009962 \\ \mathrm{H} & 1.399052 & -1.582592 & 2.080630 \\ \mathrm{C} & -0.358338 & 1.518685 & -1.509390 \\ \mathrm{C} & -0.139361 & 3.009129 & -1.400425 \\ \mathrm{C} & 0.591545 & 0.785764 & -2.436626 \\ \mathrm{C} & -1.817451 & 1.181446 & -1.811162 \\ \mathrm{~N} & -0.226866 & -1.870906 & -0.767718 \\ \mathrm{P} & -0.360939 & -3.322537 & -1.231725 \\ \mathrm{C} & -1.652041 & -3.774420 & -2.529910 \\ \mathrm{C} & 0.805415 & -4.684504 & -0.642959 \\ \mathrm{C} & -2.267707 & -5.155112 & -2.284746 \\ \mathrm{C} & -2.733138 & -2.693294 & -2.489263 \\ \mathrm{C} & -0.930546 & -3.736872 & -3.892163 \\ \mathrm{C} & 0.015626 & -5.593726 & 0.317986 \\ \mathrm{C} & 1.361955 & -5.495597 & -1.819337 \\ \mathrm{C} & 1.941348 & -3.995791 & 0.113787 \\ \mathrm{H} & -3.001627 & -5.337739 & -3.085493 \\ \mathrm{H} & -1.531579 & -5.965836 & -2.333622 \\ \mathrm{H} & -2.801937 & -5.207787 & -1.326163 \\ \mathrm{H} & 1.946221 & -4.874788 & -2.512380 \\ \mathrm{H} & 0.581948 & -6.024544 & -2.379784 \\ \mathrm{H} & 2.037918 & -6.260779 & -1.405214 \\ \mathrm{H} & 2.616106 & -4.777117 & 0.495930 \\ \mathrm{H} & 2.521555 & -3.326179 & -0.533302 \\ \mathrm{H} & 1.564532 & -3.422541 & 0.967427 \\ \mathrm{H} & 0.717694 & -6.340474 & 0.722270 \\ \mathrm{H} & -0.401263 & -5.033032 & 1.167052 \\ \mathrm{H} & -0.794629 & -6.138409 & -0.180984 \\ \mathrm{H} & -3.478866 & -2.933372 & -3.262639 \\ \mathrm{H} & -3.245614 & -2.653641 & -1.519024 \\ \mathrm{H} & -0.154077 & -4.507162 & -3.984207\end{array}$




$\begin{array}{lrrr}H & -1.683332 & -3.927814 & -4.673945 \\ H & -0.482968 & -2.752525 & -4.090375 \\ H & -2.319634 & -1.704757 & -2.713642 \\ \mathrm{C} & -1.827739 & -0.071837 & 1.114867 \\ \mathrm{H} & -2.611728 & -0.455946 & 0.440408 \\ \mathrm{H} & -1.793184 & -0.697652 & 2.017953 \\ \mathrm{H} & -2.097247 & 0.956321 & 1.418357 \\ \mathrm{H} & 0.151564 & 0.627030 & -3.437481 \\ \mathrm{H} & 1.553470 & 1.301657 & -2.539880 \\ \mathrm{H} & 0.833580 & -0.256685 & -2.097245 \\ \mathrm{H} & -2.057137 & 1.488312 & -2.847838 \\ \mathrm{H} & -2.037869 & 0.103777 & -1.758225 \\ \mathrm{H} & -2.509166 & 1.699893 & -1.134747 \\ \mathrm{H} & -0.407262 & 3.505872 & -2.352160 \\ \mathrm{H} & -0.777493 & 3.450491 & -0.621542 \\ \mathrm{H} & 0.904388 & 3.269620 & -1.182915\end{array}$

\section{$\left(\mathrm{NP}^{t} \mathrm{Bu}_{3}\right)_{2} \mathrm{TiMe}^{+}$}

$\begin{array}{rrrr}\mathrm{Ti} & -0.13384 & -0.61503 & -0.93458 \\ \mathrm{~N} & 1.50468 & -0.26339 & -0.28227 \\ \mathrm{~N} & -1.51233 & 0.44640 & -0.48255 \\ \mathrm{P} & -2.74119 & 1.42326 & -0.10424 \\ \mathrm{P} & 3.04826 & 0.09093 & 0.03114 \\ \mathrm{C} & -0.00877 & -0.68625 & -3.03114 \\ \mathrm{H} & -1.00590 & -0.89036 & -3.46018 \\ \mathrm{H} & 0.37122 & 0.24206 & -3.48698 \\ \mathrm{H} & 0.67811 & -1.50320 & -3.32192 \\ \mathrm{C} & 3.36602 & -0.12525 & 1.91333 \\ \mathrm{C} & 4.14048 & -1.12850 & -0.97678 \\ \mathrm{C} & 3.36964 & 1.90368 & -0.51389 \\ \mathrm{C} & -2.53918 & 2.01217 & 1.71365 \\ \mathrm{C} & -2.71111 & 2.90906 & -1.31293 \\ \mathrm{C} & -4.35850 & 0.41165 & -0.31682 \\ \mathrm{C} & 5.60352 & -1.17963 & -0.51370 \\ \mathrm{C} & 3.52213 & -2.53484 & -0.88574 \\ \mathrm{C} & 4.09398 & -0.73913 & -2.46298 \\ \mathrm{C} & 4.85979 & 2.24132 & -0.66199 \\ \mathrm{C} & 2.64592 & 2.15289 & -1.84850 \\ \mathrm{C} & 2.73478 & 2.86431 & 0.50406 \\ \mathrm{C} & 4.66575 & 0.53415 & 2.39540 \\ \mathrm{C} & 2.16912 & 0.46090 & 2.68037 \\ \mathrm{C} & 3.40747 & -1.62145 & 2.26413 \\ \mathrm{H} & 2.31125 & 0.23242 & 3.74839 \\ \mathrm{H} & 2.07791 & 1.54601 & 2.58338 \\ \mathrm{H} & 1.22919 & -0.00158 & 2.35333 \\ \mathrm{H} & 3.43989 & -1.70579 & 3.36184 \\ \mathrm{H} & 2.50702 & -2.14655 & 1.91848 \\ \mathrm{H} & 4.29587 & -2.13019 & 1.87419 \\ \mathrm{H} & 4.78366 & 0.30981 & 3.46783 \\ \mathrm{H} & 5.55514 & 0.14874 & 1.88233 \\ \mathrm{H} & 4.64817 & 1.62573 & 2.29506 \\ \mathrm{H} & 6.15175 & -1.85432 & -1.19218\end{array}$

\begin{tabular}{|c|c|c|c|}
\hline & & -0.20113 & \\
\hline & & & \\
\hline & 0579 & & \\
\hline & 4216 & & \\
\hline & & & \\
\hline & .58543 & 63 & \\
\hline & 3.06181 & 94 & -2.82706 \\
\hline & 286 & & \\
\hline & 4.94247 & & \\
\hline & 5.42 & & \\
\hline & & & \\
\hline & 2.7 & & \\
\hline & & & \\
\hline & & & \\
\hline & & & \\
\hline & & & \\
\hline & & & \\
\hline & & & \\
\hline & & & \\
\hline & & & \\
\hline & & & \\
\hline & & & \\
\hline & & & \\
\hline & & & \\
\hline & & & \\
\hline & & & \\
\hline & & & \\
\hline & & & \\
\hline & & & \\
\hline & & & \\
\hline & & & \\
\hline & & & \\
\hline & & & \\
\hline & & & \\
\hline & & & \\
\hline & & & \\
\hline & & & \\
\hline & & & \\
\hline & & & \\
\hline & & & \\
\hline & & & \\
\hline & & & \\
\hline & & & \\
\hline & & & \\
\hline & & & \\
\hline & & & \\
\hline & -2.2 & & \\
\hline & -3.25 & & \\
\hline & & & \\
\hline & & & \\
\hline & & & \\
\hline & & & \\
\hline & -0.1593 & 3.29096 & 1.741 \\
\hline
\end{tabular}




\section{$\left(\mathrm{NP}^{t} \mathrm{Bu}_{3}\right)_{2} \mathrm{TiMe}^{+}-\mathrm{TS}$}

\begin{tabular}{|c|c|c|c|}
\hline Гi & 0.306428 & 0.602621 & 0.280103 \\
\hline C & 2.828071 & 0.123084 & -0.533518 \\
\hline$P$ & 2.630100 & 2.039896 & 0.275285 \\
\hline $\mathrm{N}$ & -1.271607 & 0.772726 & -0.546082 \\
\hline $\mathrm{N}$ & 1.078947 & 2.240880 & 0.632747 \\
\hline$P$ & -2.718075 & 1.309931 & -1.025569 \\
\hline C & -0.277761 & -0.190037 & 2.158243 \\
\hline $\mathrm{H}$ & 0.527488 & -0.307073 & 2.900788 \\
\hline $\mathrm{H}$ & -1.026059 & 0.494403 & 2.589144 \\
\hline $\mathrm{H}$ & -0.765324 & -1.169817 & 2.003540 \\
\hline C & -2.726259 & 1.322601 & -2.948050 \\
\hline C & -4.033672 & 0.083909 & -0.354133 \\
\hline C & -2.997008 & 3.074991 & -0.328878 \\
\hline C & 3.145008 & 3.338809 & -1.041750 \\
\hline C & 3.702259 & 2.166548 & 1.859428 \\
\hline C & -5.398560 & 0.203736 & -1.045069 \\
\hline C & -3.490489 & -1.347909 & -0.511663 \\
\hline C & -4.213013 & 0.307733 & 1.156245 \\
\hline C & -4.463814 & 3.524174 & -0.387488 \\
\hline C & -2.492720 & 3.122644 & 1.125015 \\
\hline C & -2.127526 & 4.074383 & -1.108958 \\
\hline C & -3.876956 & 2.138233 & -3.552996 \\
\hline C & -1.380454 & 1.883036 & -3.439789 \\
\hline C & -2.806245 & -0.119736 & -3.473389 \\
\hline $\mathrm{H}$ & -1.358315 & 1.790339 & -4.537114 \\
\hline $\mathrm{H}$ & -1.229002 & 2.937038 & -3.191393 \\
\hline $\mathrm{H}$ & -0.546620 & 1.300181 & -3.029741 \\
\hline $\mathrm{H}$ & -2.652387 & -0.088477 & -4.563653 \\
\hline $\mathrm{H}$ & -2.020058 & -0.753948 & -3.042759 \\
\hline $\mathrm{H}$ & -3.781250 & -0.586470 & -3.297080 \\
\hline $\mathrm{H}$ & -3.828164 & 2.035637 & -4.649143 \\
\hline $\mathrm{H}$ & -4.864041 & 1.780662 & -3.235821 \\
\hline $\mathrm{H}$ & -3.801277 & 3.207976 & -3.324993 \\
\hline $\mathrm{H}$ & -6.089797 & -0.501020 & -0.555100 \\
\hline $\mathrm{H}$ & -5.835402 & 1.205943 & -0.953786 \\
\hline $\mathrm{H}$ & -5.361016 & -0.068303 & -2.106797 \\
\hline $\mathrm{H}$ & -4.192357 & -2.027695 & -0.003875 \\
\hline $\mathrm{H}$ & -3.410628 & -1.673624 & -1.552529 \\
\hline $\mathrm{H}$ & -2.509309 & -1.447898 & -0.031298 \\
\hline $\mathrm{H}$ & -4.865500 & -0.494205 & 1.536349 \\
\hline $\mathrm{H}$ & -3.257096 & 0.237707 & 1.691268 \\
\hline $\mathrm{H}$ & -4.697601 & 1.260816 & 1.395427 \\
\hline $\mathrm{H}$ & -4.512983 & 4.567647 & -0.037276 \\
\hline $\mathrm{H}$ & -4.879324 & 3.500457 & -1.402336 \\
\hline $\mathrm{H}$ & -5.111099 & 2.932911 & 0.271025 \\
\hline $\mathrm{H}$ & -2.648630 & 4.150449 & 1.490156 \\
\hline $\mathrm{H}$ & -3.029606 & 2.449821 & 1.799522 \\
\hline $\mathrm{H}$ & -1.418784 & 2.908152 & 1.180786 \\
\hline $\mathrm{H}$ & -2.190432 & 5.044016 & -0.589864 \\
\hline $\mathrm{H}$ & -1.073618 & 3.767429 & -1.109679 \\
\hline
\end{tabular}

$\begin{array}{lrrr}\mathrm{H} & -2.469619 & 4.233972 & -2.137641 \\ \mathrm{C} & 5.127662 & 1.618611 & 1.737531 \\ \mathrm{C} & 3.772234 & 3.649366 & 2.261576 \\ \mathrm{C} & 2.950548 & 1.435848 & 2.981216 \\ \mathrm{C} & 4.214706 & 0.022188 & -1.194009 \\ \mathrm{C} & 2.738786 & -0.960831 & 0.549312 \\ \mathrm{C} & 1.810923 & -0.219665 & -1.653019 \\ \mathrm{C} & 4.657861 & 3.524344 & -1.213257 \\ \mathrm{C} & 2.517150 & 2.963185 & -2.390534 \\ \mathrm{C} & 2.498490 & 4.668925 & -0.609156 \\ \mathrm{H} & 2.803116 & -1.948185 & 0.061720 \\ \mathrm{H} & 3.559599 & -0.889094 & 1.272042 \\ \mathrm{H} & 1.806065 & -0.965627 & 1.149881 \\ \mathrm{H} & 2.181024 & -1.132770 & -2.150133 \\ \mathrm{H} & 0.771593 & -0.529651 & -1.381534 \\ \mathrm{H} & 1.704383 & 0.560341 & -2.411002 \\ \mathrm{H} & 4.376821 & -1.021516 & -1.511389 \\ \mathrm{H} & 4.291340 & 0.651298 & -2.088181 \\ \mathrm{H} & 5.025655 & 0.288062 & -0.508295 \\ \mathrm{H} & 3.493224 & 1.615248 & 3.922622 \\ \mathrm{H} & 1.928112 & 1.818691 & 3.090206 \\ \mathrm{H} & 2.904696 & 0.351935 & 2.833618 \\ \mathrm{H} & 5.657712 & 1.839021 & 2.680295 \\ \mathrm{H} & 5.153506 & 0.527225 & 1.607896 \\ \mathrm{H} & 5.702301 & 2.085950 & 0.925843 \\ \mathrm{H} & 4.226571 & 3.699479 & 3.265158 \\ \mathrm{H} & 4.400770 & 4.245735 & 1.588814 \\ \mathrm{H} & 2.772634 & 4.101617 & 2.326403 \\ \mathrm{H} & 2.646368 & 3.816866 & -3.074539 \\ \mathrm{H} & 3.002628 & 2.100414 & -2.862848 \\ \mathrm{H} & 1.438132 & 2.780533 & -2.296413 \\ \mathrm{H} & 4.821435 & 4.288050 & -1.993391 \\ \mathrm{H} & 5.143495 & 3.890902 & -0.299432 \\ \mathrm{H} & 5.169846 & 2.609070 & -1.540652 \\ \mathrm{H} & 2.629981 & 5.386593 & -1.433828 \\ \mathrm{H} & 1.424776 & 4.543727 & -0.419338 \\ \mathrm{H} & 2.962778 & 5.100867 & 0.282607 \\ & & & \end{array}$

\section{$\left(\mathrm{NP}^{t} \mathrm{Bu}_{3}\right)_{2} \mathrm{TiMe}^{+}$-Product}

$\begin{array}{rrrr}\mathrm{Ti} & -0.057629 & -0.508620 & -0.124909 \\ \mathrm{~N} & 1.695547 & -0.203270 & 0.128820 \\ \mathrm{~N} & -1.119658 & 1.174604 & -0.366348 \\ \mathrm{P} & -1.928323 & 2.454071 & -0.596256 \\ \mathrm{P} & 3.274841 & 0.093427 & 0.066715 \\ \mathrm{C} & -0.165282 & -1.523461 & -1.974033 \\ \mathrm{H} & -1.221378 & -1.664110 & -2.267197 \\ \mathrm{H} & 0.348836 & -0.977824 & -2.782864 \\ \mathrm{H} & 0.302350 & -2.520646 & -1.886866 \\ \mathrm{C} & 3.896209 & 0.373503 & 1.863899 \\ \mathrm{C} & 4.149986 & -1.417948 & -0.729142 \\ \mathrm{C} & 3.561582 & 1.672534 & -0.994328 \\ \mathrm{C} & -3.214069 & 2.534160 & -1.969828\end{array}$




\begin{tabular}{|c|c|c|c|}
\hline C & -1.558401 & 4.007115 & 0.407597 \\
\hline $\mathrm{C}$ & 5.667464 & -1.445891 & -0.501841 \\
\hline C & 3.514401 & -2.701837 & -0.167242 \\
\hline$c$ & 3.864586 & -1.425738 & -2.239911 \\
\hline C & 5.011959 & 1.860685 & -1.458624 \\
\hline C & 2.625559 & 1.615837 & -2.214604 \\
\hline C & 3.133220 & 2.906824 & -0.185926 \\
\hline C & 5.281073 & 1.027272 & 1.954197 \\
\hline C & 2.863858 & 1.238238 & 2.607544 \\
\hline C & 3.932419 & -0.978158 & 2.595120 \\
\hline $\mathrm{H}$ & 3.130291 & 1.237323 & 3.676267 \\
\hline $\mathrm{H}$ & 2.851471 & 2.279733 & 2.273267 \\
\hline $\mathrm{H}$ & 1.854969 & 0.820831 & 2.503543 \\
\hline $\mathrm{H}$ & 4.132175 & -0.781072 & 3.660244 \\
\hline $\mathrm{H}$ & 2.969370 & -1.500481 & 2.525384 \\
\hline $\mathrm{H}$ & 4.727600 & -1.638911 & 2.232373 \\
\hline $\mathrm{H}$ & 5.559291 & 1.093832 & 3.018667 \\
\hline $\mathrm{H}$ & 6.060991 & 0.44 & 1.449401 \\
\hline $\mathrm{H}$ & 5.293301 & 2.048239 & 1.554155 \\
\hline $\mathrm{H}$ & 6.076163 & -2.313624 & -1.044933 \\
\hline $\mathrm{H}$ & 6.174240 & -0.552294 & -0.886601 \\
\hline $\mathrm{H}$ & 5.933325 & -1.573358 & 0.554428 \\
\hline $\mathrm{H}$ & 3.920655 & -3.551927 & -0.737555 \\
\hline $\mathrm{H}$ & 3.741621 & -2.874068 & 0.888533 \\
\hline $\mathrm{H}$ & 2.425518 & -2.690327 & -0.296617 \\
\hline $\mathrm{H}$ & 4.248508 & -2.374385 & -2.647319 \\
\hline $\mathrm{H}$ & 2.786993 & -1.387683 & -2.446163 \\
\hline $\mathrm{H}$ & 4.370360 & -0.61 & -2.776544 \\
\hline $\mathrm{H}$ & 5.078462 & 2.81 & -1.997844 \\
\hline $\mathrm{H}$ & 5.722941 & 272 & -0.624484 \\
\hline $\mathrm{H}$ & 5.336415 & 1.07 & 3744 \\
\hline $\mathrm{H}$ & 2.721280 & 2.5 & 1809 \\
\hline $\mathrm{H}$ & 2.870175 & 0.8 & 5861 \\
\hline $\mathrm{H}$ & 1.58 & 1.4 & 0460 \\
\hline $\mathrm{H}$ & 3.13 & 3.77 & 6462 \\
\hline $\mathrm{H}$ & 2.11 & 2.78 & 2897 \\
\hline $\mathrm{H}$ & 3.81 & 3.1 & 0.637429 \\
\hline C & -2.96 & 3.7 & -2.862400 \\
\hline C & -3.06 & 1.240585 & -2.772251 \\
\hline C & -4.620719 & 2.606762 & -1.350805 \\
\hline C & -0.543888 & 4.847621 & -0.383313 \\
\hline C & -2.820481 & 4.831502 & 0.680274 \\
\hline C & -0.936001 & 3.528226 & 1.722354 \\
\hline $\mathrm{H}$ & -2.528202 & 5.701537 & 1.292720 \\
\hline $\mathrm{H}$ & -3.278569 & 5.222376 & -0.238310 \\
\hline $\mathrm{H}$ & -3.575615 & 4.266081 & 1.246872 \\
\hline $\mathrm{H}$ & -0.635212 & 4.415898 & 2.300039 \\
\hline $\mathrm{H}$ & -1.650949 & 2.952800 & 2.327053 \\
\hline $\mathrm{H}$ & -0.047800 & 2.909505 & 1.547939 \\
\hline $\mathrm{H}$ & -0.277727 & 5.723156 & 0.231059 \\
\hline $\mathrm{H}$ & 0.377358 & 4.286643 & -0.590404 \\
\hline $\mathrm{H}$ & -0.955007 & 5.219166 & -1.331652 \\
\hline & -5.352748 & 2.584948 & -2.176472 \\
\hline
\end{tabular}

$\begin{array}{rrrr}H & -4.827092 & 1.743583 & -0.698942 \\ H & -4.789309 & 3.531488 & -0.782712 \\ H & -3.714463 & 3.748167 & -3.667825 \\ H & -3.072312 & 4.706480 & -2.322416 \\ H & -1.966685 & 3.724913 & -3.334212 \\ H & -3.800502 & 1.259673 & -3.587109 \\ H & -2.060172 & 1.141544 & -3.212078 \\ H & -3.252354 & 0.354778 & -2.154620 \\ \mathrm{C} & -0.714373 & -1.911424 & 1.315370 \\ \mathrm{C} & -2.085191 & -2.108367 & 0.685905 \\ \mathrm{C} & 0.001679 & -3.188387 & 1.673986 \\ \mathrm{C} & -0.753853 & -0.859925 & 2.410389 \\ \mathrm{H} & -1.605539 & -1.024123 & 3.098330 \\ \mathrm{H} & 0.175536 & -0.840919 & 2.992453 \\ \mathrm{H} & -0.922630 & 0.168913 & 2.016279 \\ \mathrm{H} & -2.845424 & -2.334037 & 1.459082 \\ \mathrm{H} & -2.462671 & -1.191412 & 0.184622 \\ \mathrm{H} & -2.086596 & -2.918997 & -0.053669 \\ \mathrm{H} & -0.543963 & -3.741253 & 2.463432 \\ \mathrm{H} & 0.084711 & -3.860799 & 0.807971 \\ \mathrm{H} & 1.013137 & -2.988934 & 2.052822\end{array}$

\section{$(\mathrm{Cp})\left(\mathrm{OSi}^{t} \mathrm{Bu}_{3}\right) \mathrm{TiMe}^{+}$}

$\begin{array}{rrrr}\mathrm{Ti} & -0.49087 & -0.03953 & -0.37811 \\ \mathrm{C} & -2.01010 & 1.70654 & 0.16066 \\ \mathrm{C} & -1.85634 & 0.96726 & 1.36340 \\ \mathrm{C} & -0.50297 & 1.04741 & 1.75727 \\ \mathrm{C} & 0.18406 & 1.84568 & 0.79760 \\ \mathrm{C} & -0.75214 & 2.26332 & -0.18246 \\ \mathrm{H} & -2.93655 & 1.83279 & -0.39829 \\ \mathrm{H} & -2.63924 & 0.41868 & 1.88102 \\ \mathrm{H} & -0.06673 & 0.61100 & 2.65008 \\ \mathrm{H} & 1.23851 & 2.11532 & 0.83000 \\ \mathrm{H} & -0.53875 & 2.89775 & -1.03740 \\ \mathrm{C} & -0.31913 & -1.75647 & 0.73432 \\ \mathrm{H} & -0.34585 & -1.84288 & 1.82455 \\ \mathrm{H} & -1.34278 & -2.02337 & 0.34821 \\ \mathrm{H} & 0.40647 & -2.45548 & 0.29475 \\ \mathrm{O} & 0.51268 & -0.25547 & -1.80144 \\ \mathrm{Si} & 0.74263 & -0.50146 & -3.51183 \\ \mathrm{C} & 0.94131 & 1.27906 & -4.25772 \\ \mathrm{C} & 2.30158 & -1.63688 & -3.62501 \\ \mathrm{C} & -0.94052 & -1.37373 & -3.98409 \\ \mathrm{C} & 1.50324 & 1.21007 & -5.69076 \\ \mathrm{C} & -0.39548 & 2.04051 & -4.30762 \\ \mathrm{C} & 1.90011 & 2.11664 & -3.38812 \\ \mathrm{C} & 2.38234 & -2.31907 & -5.00359 \\ \mathrm{C} & 3.59340 & -0.82972 & -3.40455 \\ \mathrm{C} & 2.25834 & -2.71775 & -2.52659 \\ \mathrm{C} & -1.17629 & -1.31320 & -5.50704 \\ \mathrm{C} & -0.97692 & -2.85154 & -3.56136 \\ \mathrm{C} & -2.11573 & -0.68001 & -3.27598 \\ & & & \end{array}$




$\begin{array}{rrrr}\mathrm{H} & 1.59189 & 2.23454 & -6.09111 \\ \mathrm{H} & 2.50463 & 0.76134 & -5.72640 \\ \mathrm{H} & 0.85066 & 0.64916 & -6.37469 \\ \mathrm{H} & 3.59683 & -0.30679 & -2.43732 \\ \mathrm{H} & 3.77583 & -0.09736 & -4.20148 \\ \mathrm{H} & 4.44719 & -1.52780 & -3.40303 \\ \mathrm{H} & 3.16646 & -3.33795 & -2.60921 \\ \mathrm{H} & 2.25609 & -2.27154 & -1.52204 \\ \mathrm{H} & 1.39712 & -3.39032 & -2.61420 \\ \mathrm{H} & 3.30128 & -2.92784 & -5.04675 \\ \mathrm{H} & 1.53803 & -2.99650 & -5.19035 \\ \mathrm{H} & 2.43232 & -1.59571 & -5.82978 \\ \mathrm{H} & -2.11034 & -1.84390 & -5.75815 \\ \mathrm{H} & -1.26852 & -0.28781 & -5.88654 \\ \mathrm{H} & -0.36599 & -1.80932 & -6.06003 \\ \mathrm{H} & -1.96438 & -3.27177 & -3.81825 \\ \mathrm{H} & -0.22513 & -3.45532 & -4.08392 \\ \mathrm{H} & -0.83291 & -2.98540 & -2.47921 \\ \mathrm{H} & -3.06216 & -1.18972 & -3.52821 \\ \mathrm{H} & -2.04129 & -0.77782 & -2.16405 \\ \mathrm{H} & -2.22796 & 0.38106 & -3.52330 \\ \mathrm{H} & -0.20599 & 3.06470 & -4.67158 \\ \mathrm{H} & -1.12181 & 1.58698 & -4.99393 \\ \mathrm{H} & -0.86319 & 2.13238 & -3.31594 \\ \mathrm{H} & 2.91871 & 1.71403 & -3.36461 \\ \mathrm{H} & 1.95848 & 3.13527 & -3.80741 \\ \mathrm{H} & 1.54458 & 2.20335 & -2.35105\end{array}$

\section{$(\mathrm{Cp})\left(\mathrm{OSi}^{\mathrm{t}} \mathrm{Bu}_{3}\right) \mathrm{TiMe}^{+}-$Product}

$\begin{array}{lrrr}\mathrm{Ti} & -0.280670 & -0.058871 & -0.056979 \\ \mathrm{C} & 1.939340 & -0.626289 & 0.476616 \\ \mathrm{C} & 1.939426 & 0.778864 & 0.308623 \\ \mathrm{C} & 1.129028 & 1.340776 & 1.318887 \\ \mathrm{C} & 0.625343 & 0.290030 & 2.117975 \\ \mathrm{C} & 1.123103 & -0.929296 & 1.597164 \\ \mathrm{H} & 2.468113 & -1.340667 & -0.149130 \\ \mathrm{H} & 2.478925 & 1.327728 & -0.455472 \\ \mathrm{H} & 0.907221 & 2.397227 & 1.442994 \\ \mathrm{H} & -0.012800 & 0.399984 & 2.988115 \\ \mathrm{H} & 0.917304 & -1.916706 & 1.999689 \\ \mathrm{C} & -0.283881 & 1.434724 & -1.554564 \\ \mathrm{C} & 0.076439 & 2.896840 & -1.389072 \\ \mathrm{C} & 0.568372 & 0.729550 & -2.611637 \\ \mathrm{C} & -1.773615 & 1.277443 & -1.880366 \\ \mathrm{O} & -0.714707 & -1.839938 & -0.847624 \\ \mathrm{Si} & -1.145410 & -3.301707 & -1.272389 \\ \mathrm{C} & -2.671775 & -3.499746 & -2.387610 \\ \mathrm{C} & -0.106545 & -4.794294 & -0.717908 \\ \mathrm{C} & -3.468020 & -4.774617 & -2.062682 \\ \mathrm{C} & -3.589866 & -2.275881 & -2.272780 \\ \mathrm{C} & -2.116316 & -3.589805 & -3.830661 \\ \mathrm{C} & -0.915207 & -5.503520 & 0.394090\end{array}$

$\begin{array}{rrrr}\mathrm{C} & 0.123793 & -5.771183 & -1.885572 \\ \mathrm{C} & 1.245107 & -4.337180 & -0.156586 \\ \mathrm{H} & -4.293785 & -4.854781 & -2.788580 \\ \mathrm{H} & -2.869832 & -5.689832 & -2.154258 \\ \mathrm{H} & -3.915631 & -4.742878 & -1.059257 \\ \mathrm{H} & 0.727757 & -5.327338 & -2.689998 \\ \mathrm{H} & -0.810742 & -6.153012 & -2.315777 \\ \mathrm{H} & 0.680013 & -6.640936 & -1.498109 \\ \mathrm{H} & 1.797371 & -5.226229 & 0.189077 \\ \mathrm{H} & 1.861199 & -3.836724 & -0.916246 \\ \mathrm{H} & 1.128844 & -3.663074 & 0.699351 \\ \mathrm{H} & -0.326418 & -6.368720 & 0.743081 \\ \mathrm{H} & -1.092039 & -4.856439 & 1.266982 \\ \mathrm{H} & -1.880928 & -5.889081 & 0.038966 \\ \mathrm{H} & -4.449466 & -2.422789 & -2.946666 \\ \mathrm{H} & -3.982906 & -2.139435 & -1.255641 \\ \mathrm{H} & -1.484334 & -4.475029 & -3.991025 \\ \mathrm{H} & -2.974536 & -3.671836 & -4.519089 \\ \mathrm{H} & -1.551222 & -2.690789 & -4.121313 \\ \mathrm{H} & -3.083612 & -1.354399 & -2.581634 \\ \mathrm{C} & -2.010355 & 0.209938 & 1.125989 \\ \mathrm{H} & -2.839669 & -0.028491 & 0.435802 \\ \mathrm{H} & -2.082991 & -0.437754 & 2.011596 \\ \mathrm{H} & -2.116155 & 1.258024 & 1.453581 \\ \mathrm{H} & 0.212746 & 0.992219 & -3.626184 \\ \mathrm{H} & 1.625774 & 1.013273 & -2.543201 \\ \mathrm{H} & 0.517024 & -0.373123 & -2.555150 \\ \mathrm{H} & -1.961172 & 1.610574 & -2.918834 \\ \mathrm{H} & -2.125457 & 0.232542 & -1.843139 \\ \mathrm{H} & -2.412170 & 1.871270 & -1.213082 \\ \mathrm{H} & -0.125797 & 3.450160 & -2.325715 \\ \mathrm{H} & -0.521401 & 3.375208 & -0.599974 \\ \mathrm{H} & 1.139407 & 3.042181 & -1.154070\end{array}$


3) Metalation reactions

a) Hydride transfer from $\mathrm{Cp}^{*}$ to the growing polymer $\left(\mathrm{CH}_{3}\right)$

\section{$\left(\mathrm{Cp}^{*}\right)\left(\mathrm{NC}^{t} \mathrm{Bu}_{2}\right) \mathrm{TiMe}^{+}$}

$\begin{array}{lrrr}\mathrm{Ti} & 0.113556 & -0.046854 & -0.121207 \\ \mathrm{C} & 2.433705 & 0.120993 & 0.062215 \\ \mathrm{C} & 1.928729 & 1.465630 & 0.070350 \\ \mathrm{C} & 1.120659 & 1.616009 & 1.227335 \\ \mathrm{C} & 1.128199 & 0.370268 & 1.940381 \\ \mathrm{C} & 1.961750 & -0.541380 & 1.235969 \\ \mathrm{C} & 3.422149 & -0.413020 & -0.921061 \\ \mathrm{C} & 2.312596 & 2.543106 & -0.883351 \\ \mathrm{C} & 0.433428 & 2.868203 & 1.658160 \\ \mathrm{C} & 0.442057 & 0.112187 & 3.244610 \\ \mathrm{C} & 2.325843 & -1.918055 & 1.679399 \\ \mathrm{C} & -0.600978 & 1.238434 & -1.580347 \\ \mathrm{H} & -0.576027 & 2.333238 & -1.506334 \\ \mathrm{H} & -0.097742 & 0.935319 & -2.520199 \\ \mathrm{H} & -1.658116 & 0.901115 & -1.651031 \\ \mathrm{~N} & -0.315594 & -1.816366 & -0.418404 \\ \mathrm{C} & -0.845241 & -2.903273 & -0.828257 \\ \mathrm{C} & -2.101099 & -3.408082 & -0.073054 \\ \mathrm{C} & -0.216126 & -3.614180 & -2.049095 \\ \mathrm{C} & -2.689277 & -2.278073 & 0.782975 \\ \mathrm{C} & -3.217996 & -3.913498 & -0.999514 \\ \mathrm{C} & -1.652963 & -4.535430 & 0.884062 \\ \mathrm{C} & -1.016451 & -3.171910 & -3.295771 \\ \mathrm{C} & 1.232472 & -3.146994 & -2.233988 \\ \mathrm{C} & -0.197326 & -5.146919 & -1.952325 \\ \mathrm{H} & -2.505109 & -4.800873 & 1.527344 \\ \mathrm{H} & -1.336958 & -5.440660 & 0.355250 \\ \mathrm{H} & -0.829714 & -4.198574 & 1.530981 \\ \mathrm{H} & -3.594816 & -3.110665 & -1.648480 \\ \mathrm{H} & -2.914593 & -4.758848 & -1.624501 \\ \mathrm{H} & -4.054762 & -4.255477 & -0.372798 \\ \mathrm{H} & -1.982284 & -1.942547 & 1.553722 \\ \mathrm{H} & -2.988543 & -1.420790 & 0.162107 \\ \mathrm{H} & -3.588807 & -2.646858 & 1.295315 \\ \mathrm{H} & 1.671227 & -3.670752 & -3.094333 \\ \mathrm{H} & 1.290923 & -2.069283 & -2.428895 \\ \mathrm{H} & 1.836370 & -3.378270 & -1.346446 \\ \mathrm{H} & 0.254134 & -5.540446 & -2.874707 \\ \mathrm{H} & 0.419651 & -5.487872 & -1.109563 \\ \mathrm{H} & -1.193285 & -5.591556 & -1.864443 \\ \mathrm{H} & -1.055995 & -2.075121 & -3.368977 \\ \mathrm{H} & -2.039773 & -3.562648 & -3.305596 \\ \mathrm{H} & -0.500409 & -3.553598 & -4.189266 \\ \mathrm{H} & -0.388057 & 2.669723 & 2.357795 \\ \mathrm{H} & 1.151392 & 3.522482 & 2.179940\end{array}$

$\begin{array}{rrrr}H & 0.033121 & 3.434801 & 0.807908 \\ H & 1.575503 & 3.354118 & -0.903205 \\ H & 3.276032 & 2.980537 & -0.574105 \\ H & 2.436320 & 2.167718 & -1.906387 \\ H & 4.430963 & -0.060386 & -0.649436 \\ H & 3.445364 & -1.508001 & -0.929167 \\ H & 3.223032 & -0.064559 & -1.942805 \\ H & 0.303326 & -0.959378 & 3.430951 \\ H & 1.055124 & 0.512351 & 4.068593 \\ H & -0.538601 & 0.603182 & 3.311078 \\ H & 2.570786 & -2.566389 & 0.831178 \\ H & 3.210167 & -1.874281 & 2.334754 \\ H & 1.517010 & -2.393933 & 2.246330\end{array}$

\section{$\left(\mathrm{Cp}^{*}\right)\left(\mathrm{NC}^{\mathrm{t}} \mathrm{Bu}_{2}\right) \mathrm{TiMe}^{+}-\mathrm{TS}$}

\begin{tabular}{lrrr}
$\mathrm{Ti}$ & -0.045631 & -0.270318 & -0.009531 \\
$\mathrm{C}$ & 2.332884 & -0.499059 & 0.531247 \\
$\mathrm{C}$ & 1.951162 & 0.781450 & 0.045563 \\
$\mathrm{C}$ & 0.977752 & 1.328991 & 0.967642 \\
$\mathrm{C}$ & 0.821911 & 0.386828 & 2.048983 \\
$\mathrm{C}$ & 1.629495 & -0.741908 & 1.751135 \\
$\mathrm{C}$ & 3.392608 & -1.372236 & -0.047077 \\
$\mathrm{C}$ & 2.530187 & 1.481013 & -1.136982 \\
$\mathrm{C}$ & 0.006126 & 0.584620 & 3.285147 \\
$\mathrm{C}$ & -0.211399 & 2.014831 & 0.461454 \\
$\mathrm{H}$ & -1.239021 & 0.992134 & 0.445291 \\
$\mathrm{C}$ & -2.007399 & -0.320409 & 0.854265 \\
$\mathrm{C}$ & 1.806313 & -1.941115 & 2.620345 \\
$\mathrm{~N}$ & 0.088809 & -0.638058 & -1.817860 \\
$\mathrm{C}$ & -0.132376 & -1.109220 & -2.983328 \\
$\mathrm{C}$ & 0.095385 & -2.630380 & -3.192184 \\
$\mathrm{C}$ & -0.604470 & -0.140415 & -4.092975 \\
$\mathrm{C}$ & 0.029010 & -3.358172 & -1.841701 \\
$\mathrm{C}$ & -0.917898 & -3.307528 & -4.125393 \\
$\mathrm{C}$ & 1.524638 & -2.807348 & -3.752443 \\
$\mathrm{C}$ & -2.127610 & -0.321467 & -4.276071 \\
$\mathrm{C}$ & -0.362417 & 1.310471 & -3.658897 \\
$\mathrm{C}$ & 0.118462 & -0.350071 & -5.433310 \\
$\mathrm{H}$ & 1.748122 & -3.883633 & -3.802209 \\
$\mathrm{H}$ & 1.637775 & -2.393180 & -4.760688 \\
$\mathrm{H}$ & 2.263801 & -2.335216 & -3.090247 \\
$\mathrm{H}$ & -1.936885 & -3.258631 & -3.717477 \\
$\mathrm{H}$ & -0.922616 & -2.892845 & -5.137920 \\
$\mathrm{H}$ & -0.646216 & -4.369938 & -4.211343 \\
$\mathrm{H}$ & 0.815561 & -3.011960 & -1.158436 \\
$\mathrm{H}$ & -0.954026 & -3.223790 & -1.365975 \\
$\mathrm{H}$ & 0.175387 & -4.435183 & -2.004473 \\
$\mathrm{H}$ & -0.688220 & 1.981594 & -4.465463 \\
$\mathrm{H}$ & -0.932922 & 1.560806 & -2.756031 \\
$\mathrm{H}$ & 0.701895 & 1.495405 & -3.466395 \\
$\mathrm{H}$ & -0.280878 & 0.377969 & -6.154551 \\
$\mathrm{H}$ & 1.197687 & -0.165463 & -5.337732 \\
& & & \\
\hline
\end{tabular}




$\begin{array}{lrrr}\mathrm{H} & -0.032420 & -1.346701 & -5.859457 \\ \mathrm{H} & -2.654849 & -0.202218 & -3.318076 \\ \mathrm{H} & -2.396996 & -1.289796 & -4.709871 \\ \mathrm{H} & -2.486810 & 0.464588 & -4.956890 \\ \mathrm{H} & -0.812256 & 2.549928 & 1.207872 \\ \mathrm{H} & -0.099527 & 2.597394 & -0.457003 \\ \mathrm{H} & 1.873917 & 2.280481 & -1.500044 \\ \mathrm{H} & 3.484402 & 1.948112 & -0.843606 \\ \mathrm{H} & 2.729953 & 0.790137 & -1.964111 \\ \mathrm{H} & 3.203698 & -2.434440 & 0.150297 \\ \mathrm{H} & 3.487794 & -1.232517 & -1.130029 \\ \mathrm{H} & 4.367045 & -1.124359 & 0.405617 \\ \mathrm{H} & -0.533765 & -0.322972 & 3.586225 \\ \mathrm{H} & 0.670318 & 0.863607 & 4.118506 \\ \mathrm{H} & -0.723844 & 1.394071 & 3.170125 \\ \mathrm{H} & 1.955366 & -2.857094 & 2.035057 \\ \mathrm{H} & 2.700203 & -1.809072 & 3.251905 \\ \mathrm{H} & 0.952422 & -2.092072 & 3.291521 \\ \mathrm{H} & -1.679117 & -1.272772 & 1.340236 \\ \mathrm{H} & -2.589109 & 0.225458 & 1.606917 \\ \mathrm{H} & -2.667354 & -0.577772 & 0.006875\end{array}$

\section{$\left(\mathrm{Cp}^{*} \mathrm{SiMe}_{2} \mathrm{~N}^{t} \mathrm{Bu}\right) \mathrm{TiMe}^{+}$}

$\begin{array}{lrrr}\mathrm{Ti} & 0.170477 & -0.017880 & -0.231613 \\ \mathrm{~N} & 2.026146 & 0.133677 & 0.119721 \\ \mathrm{C} & -1.604185 & 1.637994 & -0.150816 \\ \mathrm{C} & -0.353932 & 2.307060 & -0.227837 \\ \mathrm{C} & 0.437953 & 1.933611 & 0.912278 \\ \mathrm{C} & -0.377303 & 1.056319 & 1.714464 \\ \mathrm{C} & -1.623507 & 0.865989 & 1.048843 \\ \mathrm{C} & -2.745687 & 1.810773 & -1.090928 \\ \mathrm{C} & -0.017956 & 3.321721 & -1.269081 \\ \mathrm{C} & -0.153872 & 0.030939 & -2.300460 \\ \mathrm{Si} & 2.329355 & 1.707623 & 0.876206 \\ \mathrm{C} & 0.002324 & 0.430056 & 3.021312 \\ \mathrm{C} & -2.790353 & 0.064877 & 1.531813 \\ \mathrm{H} & -0.970484 & -0.731799 & -2.304069 \\ \mathrm{H} & 0.695887 & -0.383205 & -2.865102 \\ \mathrm{C} & 3.147818 & 1.690684 & 2.565030 \\ \mathrm{C} & 3.279977 & 2.875695 & -0.240177 \\ \mathrm{H} & 2.948698 & 2.652660 & 3.067674 \\ \mathrm{H} & 2.811431 & 0.887974 & 3.232628 \\ \mathrm{H} & 4.240448 & 1.606880 & 2.457795 \\ \mathrm{H} & 3.158827 & 3.921225 & 0.085991 \\ \mathrm{H} & 4.355391 & 2.644840 & -0.172437 \\ \mathrm{H} & 2.987241 & 2.797485 & -1.295975 \\ \mathrm{C} & 2.786401 & -1.082996 & -0.219057 \\ \mathrm{C} & 3.619404 & -0.865348 & -1.480232 \\ \mathrm{C} & 3.683774 & -1.480849 & 0.954651 \\ \mathrm{C} & 1.748253 & -2.201997 & -0.446205 \\ \mathrm{H} & 4.166017 & -1.782301 & -1.744788 \\ \mathrm{H} & 2.983284 & -0.578992 & -2.327922\end{array}$

$\begin{array}{rrrr}\mathrm{H} & 4.354128 & -0.065553 & -1.317817 \\ \mathrm{H} & 4.206765 & -2.421605 & 0.728097 \\ \mathrm{H} & 4.442991 & -0.709997 & 1.141812 \\ \mathrm{H} & 3.097622 & -1.624466 & 1.873041 \\ \mathrm{H} & 2.228669 & -3.159995 & -0.694596 \\ \mathrm{H} & 1.160733 & -2.393021 & 0.474524 \\ \mathrm{H} & 1.085275 & -1.995432 & -1.309619 \\ \mathrm{H} & -0.534672 & 0.909629 & -2.832842 \\ \mathrm{H} & -3.396871 & 2.624213 & -0.730716 \\ \mathrm{H} & -2.410811 & 2.082745 & -2.098710 \\ \mathrm{H} & -3.366083 & 0.908795 & -1.164543 \\ \mathrm{H} & 0.999567 & 3.702683 & -1.155652 \\ \mathrm{H} & -0.135072 & 2.938040 & -2.290227 \\ \mathrm{H} & -0.704510 & 4.176462 & -1.160604 \\ \mathrm{H} & -2.502984 & -0.702888 & 2.259940 \\ \mathrm{H} & -3.514203 & 0.731282 & 2.028059 \\ \mathrm{H} & -3.324110 & -0.427145 & 0.707740 \\ \mathrm{H} & -0.817351 & -0.172838 & 3.428866 \\ \mathrm{H} & 0.889063 & -0.214095 & 2.944027 \\ \mathrm{H} & 0.233626 & 1.213894 & 3.757142\end{array}$

\section{$\left(\mathrm{Cp}^{*} \mathrm{SiMe}_{2} \mathrm{~N}^{t} \mathrm{Bu}\right) \mathrm{TiMe}^{+}-\mathrm{TS}$}




$\begin{array}{rrrr}\text { H } & 4.123731 & -0.589484 & -1.339331 \\ \mathrm{H} & 3.945577 & -2.685390 & 0.961087 \\ \mathrm{H} & 4.255250 & -0.948366 & 1.173450 \\ \mathrm{H} & 2.899326 & -1.723013 & 2.035402 \\ \mathrm{H} & 1.919339 & -3.501852 & -0.333700 \\ \mathrm{H} & 0.903752 & -2.572210 & 0.774786 \\ \mathrm{H} & 0.781690 & -2.352179 & -1.019319 \\ \mathrm{H} & -0.680074 & 0.447602 & -3.208397 \\ \mathrm{H} & -2.695692 & 1.213309 & -1.838747 \\ \mathrm{H} & -3.207860 & 0.179992 & -0.440541 \\ \mathrm{H} & 0.734143 & 2.951600 & -2.132083 \\ \mathrm{H} & -1.011219 & 2.757499 & -2.434566 \\ \mathrm{H} & -0.422029 & 4.094825 & -1.435038 \\ \mathrm{H} & -1.954281 & -0.160940 & 2.995565 \\ \mathrm{H} & -2.993054 & 1.265261 & 2.799391 \\ \mathrm{H} & -3.139843 & -0.082327 & 1.669990 \\ \mathrm{H} & -0.284581 & 1.124338 & 3.850654 \\ \mathrm{H} & 1.144238 & 0.367408 & 3.120006 \\ \mathrm{H} & 1.122617 & 2.114389 & 3.422200\end{array}$

\section{$\left(\mathrm{Cp}^{*}\right)\left(\mathrm{NP}^{t} \mathrm{Bu}_{3}\right) \mathrm{TiMe}^{+}$}

$\begin{array}{rrrr}\mathrm{Ti} & -0.384357 & 0.082516 & -0.361600 \\ \mathrm{C} & -2.013648 & 1.675467 & 0.303416 \\ \mathrm{C} & -1.820741 & 0.838281 & 1.454584 \\ \mathrm{C} & -0.477735 & 0.981851 & 1.875698 \\ \mathrm{C} & 0.173071 & 1.901732 & 0.984157 \\ \mathrm{C} & -0.793571 & 2.361918 & 0.041607 \\ \mathrm{C} & -3.319474 & 1.909792 & -0.389805 \\ \mathrm{C} & -2.876332 & 0.030346 & 2.130332 \\ \mathrm{C} & 0.146486 & 0.348271 & 3.072312 \\ \mathrm{C} & 1.573906 & 2.408673 & 1.126678 \\ \mathrm{C} & -0.598360 & 3.464534 & -0.945020 \\ \mathrm{C} & -0.590620 & -1.867942 & 0.407774 \\ \mathrm{H} & -0.609149 & -2.005854 & 1.498167 \\ \mathrm{H} & -1.589383 & -2.165648 & 0.020694 \\ \mathrm{H} & 0.151717 & -2.551818 & -0.032923 \\ \mathrm{~N} & 0.398012 & -0.076171 & -1.962974 \\ \mathrm{P} & 0.694933 & -0.483443 & -3.509508 \\ \mathrm{C} & 0.999320 & 1.130315 & -4.499929 \\ \mathrm{C} & 2.231602 & -1.625747 & -3.583173 \\ \mathrm{C} & -0.880543 & -1.398564 & -4.120225 \\ \mathrm{C} & 1.574865 & 0.900743 & -5.903433 \\ \mathrm{C} & -0.327105 & 1.898037 & -4.616711 \\ \mathrm{C} & 1.955924 & 2.022451 & -3.686898 \\ \mathrm{C} & 2.335959 & -2.393376 & -4.909570 \\ \mathrm{C} & 3.510431 & -0.794368 & -3.390234 \\ \mathrm{C} & 2.170784 & -2.612180 & -2.404756 \\ \mathrm{C} & -0.996309 & -1.448945 & -5.650509 \\ \mathrm{C} & -0.906399 & -2.831947 & -3.566127 \\ \mathrm{C} & -2.114154 & -0.692762 & -3.531261 \\ \mathrm{H} & 1.682179 & 1.883381 & -6.390517 \\ \mathrm{H} & 2.571142 & 0.444036 & -5.880405\end{array}$

\begin{tabular}{rrrr} 
& & \\
$\mathrm{H}$ & 0.922147 & 0.291123 & -6.539845 \\
$\mathrm{H}$ & 3.472011 & -0.182144 & -2.479701 \\
$\mathrm{H}$ & 3.737062 & -0.154962 & -4.250509 \\
$\mathrm{H}$ & 4.349302 & -1.499432 & -3.281329 \\
$\mathrm{H}$ & 3.103101 & -3.198054 & -2.413283 \\
$\mathrm{H}$ & 2.113591 & -2.079671 & -1.447168 \\
$\mathrm{H}$ & 1.338095 & -3.317994 & -2.467006 \\
$\mathrm{H}$ & 3.269477 & -2.978094 & -4.888783 \\
$\mathrm{H}$ & 1.514920 & -3.104353 & -5.056181 \\
$\mathrm{H}$ & 2.387043 & -1.730918 & -5.782250 \\
$\mathrm{H}$ & -1.880494 & -2.054214 & -5.905653 \\
$\mathrm{H}$ & -1.142597 & -0.458549 & -6.097089 \\
$\mathrm{H}$ & -0.128673 & -1.924178 & -6.124114 \\
$\mathrm{H}$ & -1.893281 & -3.261867 & -3.799993 \\
$\mathrm{H}$ & -0.153759 & -3.483564 & -4.022534 \\
$\mathrm{H}$ & -0.787945 & -2.851181 & -2.475210 \\
$\mathrm{H}$ & -3.011695 & -1.195630 & -3.923856 \\
$\mathrm{H}$ & -2.141447 & -0.802284 & -2.436650 \\
$\mathrm{H}$ & -2.186654 & 0.369164 & -3.780829 \\
$\mathrm{H}$ & -0.111177 & 2.875291 & -5.076095 \\
$\mathrm{H}$ & -1.062256 & 1.395111 & -5.254871 \\
$\mathrm{H}$ & -0.767930 & 2.085394 & -3.629512 \\
$\mathrm{H}$ & 2.994362 & 1.681557 & -3.720514 \\
$\mathrm{H}$ & 1.925818 & 3.033073 & -4.122616 \\
$\mathrm{H}$ & 1.640782 & 2.089586 & -2.638562 \\
$\mathrm{H}$ & 1.180113 & 0.029124 & 2.884645 \\
$\mathrm{H}$ & 0.175114 & 1.070745 & 3.904054 \\
$\mathrm{H}$ & -0.422475 & -0.524007 & 3.413776 \\
$\mathrm{H}$ & 1.977622 & 2.769956 & 0.172650 \\
$\mathrm{H}$ & 1.596869 & 3.251660 & 1.836465 \\
$\mathrm{H}$ & 2.254611 & 1.639777 & 1.515594 \\
$\mathrm{H}$ & -2.473451 & -0.890195 & 2.569402 \\
$\mathrm{H}$ & -3.332290 & 0.614745 & 2.945398 \\
$\mathrm{H}$ & -3.682783 & -0.249249 & 1.440942 \\
$\mathrm{H}$ & -3.884996 & 2.692957 & 0.141656 \\
$\mathrm{H}$ & -3.184859 & 2.250750 & -1.423922 \\
$\mathrm{H}$ & -3.951265 & 1.012004 & -0.406274 \\
$\mathrm{H}$ & 0.417582 & 3.482700 & -1.355148 \\
$\mathrm{H}$ & -1.303677 & 3.399437 & -1.781560 \\
& -0.770149 & 4.434420 & -0.449314 \\
& & & \\
\hline
\end{tabular}

\section{$\left(\mathrm{Cp}^{*}\right)\left(\mathrm{NP}^{\mathrm{t}} \mathrm{Bu}_{3}\right) \mathrm{TiMe}^{+}-\mathrm{TS}$}

$\begin{array}{lrrr}\mathrm{Ti} & 0.006203 & 0.069423 & -0.006411 \\ \mathrm{C} & 2.451591 & -0.191401 & 0.041325 \\ \mathrm{C} & 1.976607 & 1.145398 & -0.055747 \\ \mathrm{C} & 1.224846 & 1.431191 & 1.151447 \\ \mathrm{C} & 1.303749 & 0.267158 & 1.998243 \\ \mathrm{C} & 2.025396 & -0.726301 & 1.296187 \\ \mathrm{C} & 3.389607 & -0.870009 & -0.899136 \\ \mathrm{C} & 2.302530 & 2.129850 & -1.131584 \\ \mathrm{C} & -0.043532 & 2.150537 & 1.066002 \\ \mathrm{H} & -1.059117 & 1.071857 & 1.044487\end{array}$




\begin{tabular}{|c|c|c|c|c|c|c|c|}
\hline C & -1.655717 & -0.300968 & 1.369320 & $\mathrm{H}$ & 3.222238 & 2.674326 & -0.861104 \\
\hline C & 0.797054 & 0.156422 & 3.399348 & $\mathrm{H}$ & 2.476279 & 1.638230 & -2.095284 \\
\hline C & 2.395500 & -2.073489 & 1.819687 & H & 4.420584 & -0.793614 & -0.515964 \\
\hline $\mathrm{H}$ & -2.643066 & -0.272821 & 0.891856 & $\mathrm{H}$ & 3.161179 & -1.938071 & -1.008984 \\
\hline $\mathrm{H}$ & -1.257202 & -1.343073 & 1.269351 & $\mathrm{H}$ & 3.376737 & -0.412953 & -1.894831 \\
\hline $\mathrm{H}$ & -1.756904 & -0.114880 & 2.445471 & $\mathrm{H}$ & 1.701355 & -2.417013 & 2.596148 \\
\hline $\mathrm{N}$ & -0.671916 & 0.029743 & -1.672097 & $\mathrm{H}$ & 2.428107 & -2.829042 & 1.024425 \\
\hline$P$ & -1.432004 & 0.003554 & -3.110667 & $\mathrm{H}$ & 3.400113 & -2.034328 & 2.272293 \\
\hline C & -0.126452 & 0.133042 & -4.510702 & & & & \\
\hline C & -2.384748 & -1.661137 & -3.230373 & \multirow{2}{*}{\multicolumn{4}{|c|}{$\left(\mathrm{Cp}^{*}\right)\left(\mathrm{OSi}^{\mathrm{t}} \mathrm{Bu}_{3}\right) \mathrm{TiMe}^{+}$}} \\
\hline C & -2.641379 & 1.494304 & -3.158146 & & & & \\
\hline C & -0.663486 & -0.273478 & -5.889958 & $\mathrm{Ti}$ & -0.503210 & 0.074251 & -0.472328 \\
\hline C & 0.396874 & 1.576146 & -4.590154 & C & -2.078637 & 1.686753 & 0.275381 \\
\hline C & 1.073514 & -0.752880 & -4.137032 & C & -1.884806 & 0.824351 & 1.398187 \\
\hline C & -3.465042 & -1.666169 & -4.321328 & C & -0.520013 & 0.899388 & 1.781199 \\
\hline C & -1.391775 & -2.803250 & -3.499780 & C & 0.137185 & 1.817537 & 0.892142 \\
\hline C & -3.028827 & -1.966963 & -1.867533 & C & -0.837032 & 2.328844 & -0.015922 \\
\hline C & -3.168893 & 1.806497 & -4.565249 & C & -3.386827 & 1.975510 & -0.388520 \\
\hline C & -3.829933 & 1.212789 & -2.225168 & C & -2.951595 & 0.037991 & 2.080570 \\
\hline C & -1.927044 & 2.731341 & -2.586100 & C & 0.100486 & 0.284903 & 2.987766 \\
\hline $\mathrm{H}$ & 0.142708 & -0.117986 & -6.625078 & C & 1.560877 & 2.259547 & 1.002962 \\
\hline $\mathrm{H}$ & -0.944619 & -1.331621 & -5.940749 & C & -0.637894 & 3.445731 & -0.984827 \\
\hline $\mathrm{H}$ & -1.518289 & 0.334111 & -6.210941 & C & -0.431292 & -1.784481 & 0.441744 \\
\hline $\mathrm{H}$ & -0.572672 & -2.812597 & -2.768547 & $\mathrm{H}$ & -0.431320 & -1.963886 & 1.521734 \\
\hline $\mathrm{H}$ & -0.973398 & -2.778836 & -4.511735 & $\mathrm{H}$ & -1.440213 & -2.065265 & 0.048179 \\
\hline $\mathrm{H}$ & -1.940175 & -3.752758 & -3.397574 & $\mathrm{H}$ & 0.315427 & -2.421201 & -0.054679 \\
\hline $\mathrm{H}$ & -3.517316 & -2.950861 & -1.944150 & 0 & 0.585335 & -0.054759 & -1.872308 \\
\hline $\mathrm{H}$ & -2.271113 & -2.028365 & -1.077574 & $\mathrm{Si}$ & 0.800683 & -0.488573 & -3.532912 \\
\hline $\mathrm{H}$ & -3.791756 & -1.242225 & -1.568999 & C & 1.028120 & 1.195413 & -4.474801 \\
\hline $\mathrm{H}$ & -3.899607 & -2.677762 & -4.362592 & C & 2.337221 & -1.663372 & -3.603907 \\
\hline $\mathrm{H}$ & -4.285857 & -0.972434 & -4.104615 & C & -0.910385 & -1.368368 & -3.899329 \\
\hline $\mathrm{H}$ & -3.067977 & -1.441485 & -5.318344 & C & 1.558629 & 0.982486 & -5.904252 \\
\hline $\mathrm{H}$ & -3.893015 & 2.632495 & -4.479501 & C & -0.293154 & 1.979220 & -4.568907 \\
\hline $\mathrm{H}$ & -2.378557 & 2.141744 & -5.247071 & C & 2.021253 & 2.089639 & -3.706082 \\
\hline $\mathrm{H}$ & -3.693286 & 0.959469 & -5.023249 & C & 2.377545 & -2.430593 & -4.939543 \\
\hline $\mathrm{H}$ & -4.427488 & 2.135292 & -2.156779 & C & 3.649111 & -0.870027 & -3.462173 \\
\hline $\mathrm{H}$ & -4.492098 & 0.424291 & -2.598526 & C & 2.304113 & -2.677425 & -2.444240 \\
\hline $\mathrm{H}$ & -3.495746 & 0.959171 & -1.210701 & C & -1.233116 & -1.386455 & -5.405808 \\
\hline $\mathrm{H}$ & -2.670871 & 3.539702 & -2.510018 & C & -0.953989 & -2.823553 & -3.402938 \\
\hline $\mathrm{H}$ & -1.540574 & 2.528188 & -1.579782 & C & -2.034742 & -0.614046 & -3.165421 \\
\hline $\mathrm{H}$ & -1.109516 & 3.093361 & -3.215492 & $\mathrm{H}$ & 1.653296 & 1.962424 & -6.403003 \\
\hline $\mathrm{H}$ & 1.247951 & 1.585116 & -5.289169 & $\mathrm{H}$ & 2.553214 & 0.517594 & -5.915105 \\
\hline $\mathrm{H}$ & -0.347733 & 2.279245 & -4.979174 & $\mathrm{H}$ & 0.885261 & 0.367918 & -6.518728 \\
\hline $\mathrm{H}$ & 0.758688 & 1.934122 & -3.618643 & $\mathrm{H}$ & 3.682223 & -0.284047 & -2.532189 \\
\hline $\mathrm{H}$ & 0.858140 & -1.823335 & -4.201566 & $\mathrm{H}$ & 3.828762 & -0.195616 & -4.308824 \\
\hline $\mathrm{H}$ & 1.886428 & -0.535404 & -4.847382 & $\mathrm{H}$ & 4.490292 & -1.583129 & -3.432024 \\
\hline $\mathrm{H}$ & 1.427972 & -0.529462 & -3.123431 & $\mathrm{H}$ & 3.214625 & -3.298583 & -2.493948 \\
\hline $\mathrm{H}$ & -0.491739 & 2.460876 & 2.017927 & $\mathrm{H}$ & 2.303861 & -2.174103 & -1.467044 \\
\hline $\mathrm{H}$ & -0.119619 & 2.942284 & 0.314548 & $\mathrm{H}$ & 1.446244 & -3.357774 & -2.491318 \\
\hline $\mathrm{H}$ & 0.307840 & -0.806918 & 3.597472 & $\mathrm{H}$ & 3.277572 & -3.068922 & -4.958466 \\
\hline $\mathrm{H}$ & 1.639684 & 0.247185 & 4.102749 & $\mathrm{H}$ & 1.510987 & -3.092229 & -5.073976 \\
\hline $\mathrm{H}$ & 0.088796 & 0.957021 & 3.642963 & $\mathrm{H}$ & 2.436292 & -1.761470 & -5.809227 \\
\hline $\mathrm{H}$ & 1.509237 & 2.876722 & -1.262787 & $\mathrm{H}$ & -2.187709 & -1.914075 & -5.573224 \\
\hline
\end{tabular}




$\begin{array}{rrrr}\mathrm{H} & -1.331226 & -0.383746 & -5.838947 \\ \mathrm{H} & -0.462468 & -1.927639 & -5.973241 \\ \mathrm{H} & -1.965764 & -3.231327 & -3.572697 \\ \mathrm{H} & -0.253188 & -3.468228 & -3.947612 \\ \mathrm{H} & -0.737533 & -2.913622 & -2.329595 \\ \mathrm{H} & -3.018372 & -1.047541 & -3.420230 \\ \mathrm{H} & -1.971801 & -0.766988 & -2.058216 \\ \mathrm{H} & -2.075230 & 0.460273 & -3.378589 \\ \mathrm{H} & -0.096086 & 2.955504 & -5.044282 \\ \mathrm{H} & -1.052130 & 1.473121 & -5.178597 \\ \mathrm{H} & -0.718982 & 2.186179 & -3.577468 \\ \mathrm{H} & 3.037772 & 1.681244 & -3.686967 \\ \mathrm{H} & 2.070185 & 3.075277 & -4.199531 \\ \mathrm{H} & 1.701007 & 2.249788 & -2.667368 \\ \mathrm{H} & 1.148486 & 0.008117 & 2.818922 \\ \mathrm{H} & 0.080610 & 1.012566 & 3.815674 \\ \mathrm{H} & -0.439890 & -0.606456 & 3.324354 \\ \mathrm{H} & 1.945694 & 2.649405 & 0.053222 \\ \mathrm{H} & 1.637860 & 3.066372 & 1.750242 \\ \mathrm{H} & 2.221763 & 1.447292 & 1.331492 \\ \mathrm{H} & -2.538708 & -0.788930 & 2.668684 \\ \mathrm{H} & -3.507480 & 0.691177 & 2.772624 \\ \mathrm{H} & -3.681581 & -0.375658 & 1.373042 \\ \mathrm{H} & -3.884325 & 2.811006 & 0.131413 \\ \mathrm{H} & -3.264795 & 2.275469 & -1.437061 \\ \mathrm{H} & -4.070820 & 1.117928 & -0.352141 \\ \mathrm{H} & 0.391355 & 3.499154 & -1.355943 \\ \mathrm{H} & -1.309610 & 3.377090 & -1.848183 \\ \mathrm{H} & -0.856794 & 4.401414 & -0.479445 \\ & & & \end{array}$

b) Hydride transfer from the ancillary ligand to the growing polymer $\left(\mathrm{CH}_{3}\right)$

\section{$\left(\mathrm{NP}^{\mathrm{t}} \mathrm{Bu}_{3}\right)_{2} \mathrm{TiMe}^{+}-\mathrm{TS}$}

$\begin{array}{rrrr}\mathrm{Ti} & 0.420899 & -0.124964 & -0.545982 \\ \mathrm{H} & 2.186391 & 0.079254 & -0.916364 \\ \mathrm{~N} & -0.136581 & 1.467216 & 0.126550 \\ \mathrm{~N} & -0.719357 & -1.191168 & -1.462603 \\ \mathrm{P} & -1.978692 & -2.075398 & -1.952652 \\ \mathrm{P} & 0.529284 & 2.882730 & -0.295535 \\ \mathrm{C} & 1.887287 & 1.061041 & -1.927527 \\ \mathrm{H} & 1.691199 & 0.535441 & -2.878872 \\ \mathrm{H} & 2.986970 & 1.153363 & -1.881505 \\ \mathrm{C} & 2.354851 & -1.045185 & 0.000756 \\ \mathrm{H} & 1.789528 & -1.163124 & 0.958472 \\ \mathrm{H} & 2.343573 & -2.002655 & -0.540535 \\ \mathrm{H} & 3.385063 & -0.805927 & 0.289254 \\ \mathrm{C} & -0.806122 & 4.247774 & -0.367845 \\ \mathrm{C} & 1.891583 & 3.355018 & 0.963688 \\ \mathrm{C} & 1.283308 & 2.508995 & -2.018336 \\ \mathrm{C} & -1.775776 & -2.344444 & -3.841106 \\ \mathrm{C} & -1.910719 & -3.752927 & -1.015470\end{array}$

\begin{tabular}{|c|c|c|c|}
\hline $\mathrm{C}$ & -3.623542 & -1.160860 & -1.568992 \\
\hline & 2.386346 & 4.801196 & 0.829778 \\
\hline & 1.350311 & 3.106345 & 2.383585 \\
\hline 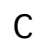 & 3.081153 & 2.402300 & 0.780509 \\
\hline C & 2.334567 & 3.507498 & -2.513758 \\
\hline C & 0.158345 & 2.450727 & -3.064562 \\
\hline C & -0.380500 & 5.466463 & -1.197822 \\
\hline C & -2.090917 & 3.634107 & -0.949324 \\
\hline C & -1.160111 & 4.700057 & 1.058180 \\
\hline $\mathrm{H}$ & -2.879822 & 4.401132 & -0.900601 \\
\hline $\mathrm{H}$ & -1.995018 & 3.325466 & -1.994188 \\
\hline $\mathrm{H}$ & -2.412437 & 2.770731 & -0.353783 \\
\hline $\mathrm{H}$ & -2.042700 & 5.355527 & 0.990869 \\
\hline $\mathrm{H}$ & -1.427872 & 3.849743 & 1.699942 \\
\hline $\mathrm{H}$ & -0.362392 & 5.279107 & 1.536032 \\
\hline $\mathrm{H}$ & -1.186641 & 6.215626 & -1.144383 \\
\hline $\mathrm{H}$ & 0.532704 & 2354 & -0.820135 \\
\hline $\mathrm{H}$ & -0.235465 & 24991 & -2.258065 \\
\hline $\mathrm{H}$ & 3.195071 & 6173 & 1.562169 \\
\hline $\mathrm{H}$ & 2.798634 & 317 & -0.163682 \\
\hline $\mathrm{H}$ & 1.604607 & 5722 & 1.053894 \\
\hline $\mathrm{H}$ & 2.199170 & 4720 & 3.083371 \\
\hline $\mathrm{H}$ & 0.618165 & 3689 & 2.701903 \\
\hline $\mathrm{H}$ & 0.892866 & 135 & 2.467354 \\
\hline $\mathrm{H}$ & 3.824782 & 408 & 1.558722 \\
\hline $\mathrm{H}$ & 2.779774 & 794 & 24334 \\
\hline $\mathrm{H}$ & 3.579397 & 317 & 89395 \\
\hline $\mathrm{H}$ & 2.690692 & 249 & -3.502524 \\
\hline $\mathrm{H}$ & 1.919390 & 352 & -2.634982 \\
\hline $\mathrm{H}$ & 3.208412 & 418 & 54808 \\
\hline $\mathrm{H}$ & 0.602195 & 976 & -4.014097 \\
\hline $\mathrm{H}$ & -0.627316 & 277 & -2.784575 \\
\hline $\mathrm{H}$ & -0.296901 & 716 & -3.249066 \\
\hline C & -3.216931 & -4.5 & -1.096978 \\
\hline C & -0.764326 & 1775 & -1.574276 \\
\hline C & -1.560899 & -3.4 & 0.458029 \\
\hline C & -4.835837 & 9433 & -2.335018 \\
\hline C & -3.911827 & 7761 & -0.060068 \\
\hline C & -3.428491 & 8664 & -1.897620 \\
\hline C & -2.637222 & -3.4 & -4.396979 \\
\hline C & -2.131745 & 5429 & -4.581701 \\
\hline C & -0.290976 & -2.618614 & -4.139524 \\
\hline $\mathrm{H}$ & -4.777899 & -0.577993 & 0.142796 \\
\hline $\mathrm{H}$ & -4.174636 & -2.233505 & 0.285557 \\
\hline $\mathrm{H}$ & -3.069392 & -0.844820 & 0.532363 \\
\hline $\mathrm{H}$ & -4.323738 & 0.868828 & -1.550651 \\
\hline $\mathrm{H}$ & -2.558900 & 0.733329 & -1.366199 \\
\hline $\mathrm{H}$ & -3.318005 & 0.527311 & -2.967773 \\
\hline $\mathrm{H}$ & -5.727449 & -1.146861 & -2.008718 \\
\hline $\mathrm{H}$ & -4.746947 & -1.570412 & -3.420385 \\
\hline $\mathrm{H}$ & -5.027417 & -2.771341 & -2.130777 \\
\hline $\mathrm{H}$ & -1.466105 & -4.455524 & 0.965084 \\
\hline & -0.592701 & -2.970173 & 0.541305 \\
\hline
\end{tabular}




$\begin{array}{lrrr}H & -2.316950 & -2.901686 & 0.993412 \\ H & -3.054235 & -5.525018 & -0.591440 \\ H & -4.052907 & -4.063171 & -0.585075 \\ H & -3.518158 & -4.776517 & -2.130395 \\ H & -0.644338 & -5.485454 & -0.912156 \\ H & -0.965487 & -4.993741 & -2.582459 \\ H & 0.187841 & -4.063332 & -1.578280 \\ H & -1.863476 & -1.180590 & -5.641394 \\ H & -3.201192 & -0.810725 & -4.545211 \\ H & -1.558195 & -0.191106 & -4.199214 \\ H & -2.491583 & -3.524182 & -5.490662 \\ H & -2.343541 & -4.469474 & -4.001431 \\ H & -3.710643 & -3.342928 & -4.215285 \\ H & -0.167362 & -2.635170 & -5.233653 \\ H & 0.344594 & -1.822459 & -3.731557 \\ H & 0.059727 & -3.579600 & -3.753229\end{array}$

\section{$\left(\mathrm{Cp}^{*}\right)\left(\mathrm{OSi}^{\mathrm{t}} \mathrm{Bu}_{3}\right) \mathrm{TiMe}^{+}-\mathrm{TS}$}

$\begin{array}{lrrr}\mathrm{Ti} & 0.037877 & -0.048078 & 0.001561 \\ \mathrm{H} & 1.822507 & -0.085113 & -0.069025 \\ \mathrm{C} & -0.542027 & 2.276381 & 0.109332 \\ \mathrm{C} & -0.003152 & 2.120630 & -1.187940 \\ \mathrm{C} & -0.814434 & 1.205936 & -1.894503 \\ \mathrm{C} & -1.874864 & 0.802436 & -1.038314 \\ \mathrm{C} & -1.709405 & 1.467095 & 0.202512 \\ \mathrm{H} & -0.157073 & 2.932871 & 0.883923 \\ \mathrm{H} & 0.889316 & 2.610166 & -1.567655 \\ \mathrm{H} & -0.667020 & 0.876723 & -2.920158 \\ \mathrm{H} & -2.674690 & 0.107439 & -1.287504 \\ \mathrm{H} & -2.373519 & 1.382486 & 1.059536 \\ \mathrm{C} & 1.879070 & 0.417490 & 1.310635 \\ \mathrm{H} & 2.050949 & 1.505823 & 1.273496 \\ \mathrm{H} & 2.870056 & -0.053653 & 1.190662 \\ \mathrm{C} & 1.673519 & -0.643412 & -1.365532 \\ \mathrm{H} & 2.034034 & 0.100927 & -2.082695 \\ \mathrm{H} & 2.437972 & -1.410550 & -1.195632 \\ \mathrm{H} & 0.794238 & -1.168955 & -1.808030 \\ \mathrm{O} & -0.306514 & -1.505238 & 0.947960 \\ \mathrm{Si} & 0.378966 & -1.736266 & 2.539815 \\ \mathrm{C} & -1.094329 & -1.988678 & 3.769081 \\ \mathrm{C} & 1.559169 & -3.255571 & 2.329568 \\ \mathrm{C} & 1.303497 & -0.007526 & 2.705687 \\ \mathrm{C} & -0.632176 & -1.764548 & 5.222038 \\ \mathrm{C} & -2.255471 & -1.025806 & 3.453649 \\ \mathrm{C} & -1.659274 & -3.417295 & 3.661039 \\ \mathrm{C} & 2.055271 & -3.761295 & 3.696330 \\ \mathrm{C} & 0.823092 & -4.397334 & 1.600196 \\ \mathrm{C} & 2.781015 & -2.899268 & 1.467056 \\ \mathrm{C} & 2.483387 & -0.059649 & 3.697551 \\ \mathrm{C} & 0.369701 & 1.110004 & 3.211968 \\ \mathrm{H} & -1.483774 & -1.948971 & 5.898879 \\ \mathrm{H} & 0.172292 & -2.451840 & 5.518864\end{array}$

$\begin{array}{rrrr}H & -0.291279 & -0.737126 & 5.407712 \\ H & 0.422188 & -4.073884 & 0.628571 \\ H & -0.000089 & -4.818650 & 2.186841 \\ H & 1.541155 & -5.212557 & 1.410156 \\ H & 3.410586 & -3.798060 & 1.354717 \\ H & 2.482205 & -2.593974 & 0.456465 \\ H & 3.413910 & -2.118578 & 1.907106 \\ H & 2.734956 & -4.615607 & 3.536427 \\ H & 2.616972 & -2.997166 & 4.253250 \\ H & 1.234483 & -4.117049 & 4.332919 \\ H & 2.974203 & 0.926989 & 3.741095 \\ H & 2.132045 & -0.296777 & 4.712766 \\ H & 3.244001 & -0.799024 & 3.419572 \\ H & 0.915032 & 2.069627 & 3.201690 \\ H & -0.541985 & 1.237439 & 2.614935 \\ H & 0.054838 & 0.928691 & 4.247277 \\ H & -3.097165 & -1.260000 & 4.127088 \\ H & -1.997173 & 0.026385 & 3.620482 \\ H & -2.618837 & -1.149134 & 2.422746 \\ H & -0.938399 & -4.180546 & 3.979053 \\ H & -2.531852 & -3.497853 & 4.330856 \\ H & -2.002411 & -3.656770 & 2.644053\end{array}$

\section{$(\mathrm{Cp})\left(\mathrm{NC}^{t} \mathrm{Bu}_{2}\right) \mathrm{TiMe}^{+}-\mathrm{TS}$}

$\begin{array}{rrrr}\mathrm{Ti} & 0.33688 & -0.22620 & -0.06640 \\ \mathrm{C} & 2.53090 & 0.17212 & 0.55803 \\ \mathrm{C} & 2.08459 & 1.38683 & -0.03657 \\ \mathrm{C} & 1.09341 & 1.94199 & 0.80293 \\ \mathrm{C} & 0.91502 & 1.07642 & 1.90814 \\ \mathrm{C} & 1.80703 & -0.01689 & 1.76302 \\ \mathrm{H} & 3.30728 & -0.48668 & 0.17209 \\ \mathrm{H} & 2.47253 & 1.83193 & -0.94926 \\ \mathrm{H} & 0.57007 & 2.87809 & 0.63387 \\ \mathrm{H} & 0.22619 & 1.22787 & 2.73576 \\ \mathrm{H} & 1.92490 & -0.84780 & 2.45644 \\ \mathrm{C} & 0.11800 & 0.07072 & -2.25793 \\ \mathrm{H} & 1.21279 & -0.11572 & -2.14447 \\ \mathrm{H} & -0.82516 & 0.54222 & -1.19200 \\ \mathrm{C} & -1.66286 & 0.71161 & -0.04629 \\ \mathrm{H} & 0.03680 & 0.98625 & -2.86177 \\ \mathrm{H} & -2.55216 & 0.38496 & -0.59742 \\ \mathrm{H} & -1.74355 & 1.77532 & 0.19813 \\ \mathrm{H} & -1.66051 & 0.13019 & 0.90764 \\ \mathrm{~N} & -0.18449 & -1.87185 & -0.71940 \\ \mathrm{C} & -0.44358 & -2.29209 & -1.90705 \\ \mathrm{C} & -0.60782 & -3.78586 & -2.17016 \\ \mathrm{C} & -0.52002 & -1.15211 & -2.95183 \\ \mathrm{C} & -0.95103 & -4.48818 & -0.84897 \\ \mathrm{C} & -1.69388 & -4.11292 & -3.20323 \\ \mathrm{C} & 0.75909 & -4.32116 & -2.66116 \\ \mathrm{C} & -1.99521 & -0.85614 & -3.29197 \\ \mathrm{C} & 0.25209 & -1.42627 & -4.25201\end{array}$




$\begin{array}{rrrr}\mathrm{H} & 0.68404 & -5.41568 & -2.74144 \\ \mathrm{H} & 1.03785 & -3.92949 & -3.64653 \\ \mathrm{H} & 1.55511 & -4.08678 & -1.93993 \\ \mathrm{H} & -2.68351 & -3.77010 & -2.86986 \\ \mathrm{H} & -1.48439 & -3.69569 & -4.19482 \\ \mathrm{H} & -1.74245 & -5.20579 & -3.31450 \\ \mathrm{H} & -0.16045 & -4.34173 & -0.10320 \\ \mathrm{H} & -1.89394 & -4.10946 & -0.43164 \\ \mathrm{H} & -1.06156 & -5.56541 & -1.03234 \\ \mathrm{H} & 0.18835 & -0.53411 & -4.89256 \\ \mathrm{H} & 1.31410 & -1.63071 & -4.05922 \\ \mathrm{H} & -0.17352 & -2.26522 & -4.81489 \\ \mathrm{H} & -2.62017 & -0.77715 & -2.39320 \\ \mathrm{H} & -2.42180 & -1.63599 & -3.93278 \\ \mathrm{H} & -2.04854 & 0.09569 & -3.84104\end{array}$

$\begin{array}{lllr}H & 0.759487 & -2.971908 & -0.933746 \\ H & 0.476777 & -2.279379 & 0.686550\end{array}$

\section{$\left(\mathrm{CpSiMe}_{2} \mathrm{~N}^{t} \mathrm{Bu}\right) \mathrm{TiMe}^{+}-\mathrm{TS}$}

$\begin{array}{lrrr}\mathrm{Ti} & 0.050648 & -0.029833 & 0.032738 \\ \mathrm{~N} & 1.922504 & 0.032498 & -0.017241 \\ \mathrm{C} & -1.451385 & 1.845574 & -0.002028 \\ \mathrm{C} & -0.155061 & 2.306336 & -0.327059 \\ \mathrm{C} & 0.717401 & 2.079096 & 0.781568 \\ \mathrm{C} & -0.083564 & 1.479396 & 1.800437 \\ \mathrm{C} & -1.409167 & 1.328348 & 1.318201 \\ \mathrm{H} & -2.331299 & 1.898024 & -0.637591 \\ \mathrm{H} & 0.138979 & 2.735646 & -1.282029 \\ \mathrm{C} & -0.680017 & -0.373201 & -2.111922 \\ \mathrm{H} & -0.006531 & -1.267840 & -1.299410 \\ \mathrm{Si} & 2.536098 & 1.600130 & 0.554437 \\ \mathrm{H} & 0.274325 & 1.154778 & 2.776949 \\ \mathrm{H} & -2.253622 & 0.920047 & 1.872195 \\ \mathrm{H} & -1.762288 & -0.286001 & -1.930445 \\ \mathrm{H} & -0.539735 & -1.162754 & -2.865652 \\ \mathrm{H} & -0.303579 & 0.560997 & -2.547515 \\ \mathrm{C} & 3.484149 & 1.502438 & 2.160870 \\ \mathrm{C} & 3.402613 & 2.587380 & -0.769772 \\ \mathrm{H} & 3.648277 & 2.518268 & 2.555352 \\ \mathrm{H} & 2.955976 & 0.921864 & 2.930876 \\ \mathrm{H} & 4.474945 & 1.045676 & 2.013656 \\ \mathrm{H} & 3.548877 & 3.625340 & -0.429408 \\ \mathrm{H} & 4.397744 & 2.171353 & -0.990080 \\ \mathrm{H} & 2.832372 & 2.612950 & -1.709268 \\ \mathrm{C} & 2.229264 & -1.367752 & -0.334310 \\ \mathrm{C} & 2.864881 & -1.483268 & -1.717114 \\ \mathrm{C} & 3.124155 & -1.990597 & 0.738152 \\ \mathrm{C} & 0.826355 & -2.046371 & -0.350184 \\ \mathrm{H} & 3.063846 & -2.536793 & -1.959427 \\ \mathrm{H} & 2.211748 & -1.058671 & -2.490544 \\ \mathrm{H} & 3.819474 & -0.940710 & -1.737297 \\ \mathrm{H} & 3.244366 & -3.066334 & 0.547234 \\ \mathrm{H} & 4.119936 & -1.525771 & 0.720720 \\ \mathrm{H} & 2.692136 & -1.860064 & 1.739476\end{array}$

